\title{
Para-selective dehydrogenative phenothiazination of hydroquinolines and indolines
}

Pooja Y. Vemuri, Yongchao Wang, and Frederic W. Patureau*

Frederic.Patureau@rwth-aachen.de

RWTH Aachen University, Institute of Organic Chemistry, Landoltweg 1, 52074 Aachen, Germany 
Substrates were purchased either from Sigma Aldrich, Acros, $A B C R$, chemPUR, or $\mathrm{TCl}$, and engaged directly. Flash chromatography was performed on silica gel (40-63 mesh) by standard technique. The IR spectra were recorded on PerkinElmer Spectrum 100 spectrometer with an attached UATR device Diamond KRS-5. All IR data were collected by attenuated total reflectance (ATR) and wave numbers $v$ are given in $\mathrm{cm}^{-1}$. High resolution mass spectra (HRMS) were obtained on a Thermo Scientific LTQ Orbitrap XL spectrometer. NMR spectra were obtained on a VNMRS 300 or VNMRS 400 or VNMRS 600 using $\mathrm{CDCl}_{3}$ or DMSO- $d_{6}$ as solvents. Coupling constants $(\mathrm{J})$ are quoted in $\mathrm{Hz}$.

\section{General experimental method for hydroquinolines and indolines}<smiles>[R]c1ccc([X])c(Nc2ccccc2[X])c1</smiles>

$1(0.5 \mathrm{mmol})$

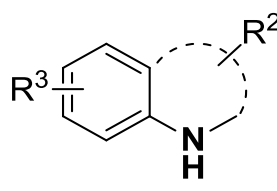

$2(0.5 \mathrm{mmol})$

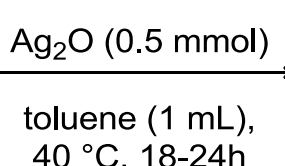
$40{ }^{\circ} \mathrm{C}, 18-24 \mathrm{~h}$

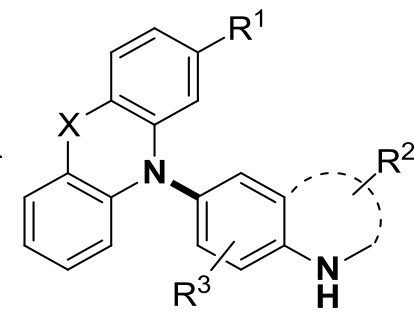

3

Unless otherwise specified, a $20 \mathrm{~mL}$ reaction vial is filled with phenothiazine $(0.5 \mathrm{mmol}), 1,2,3,4-$ tetrahydroquinoline (or indoline) $(0.5 \mathrm{mmol})$, silver oxide $(0.5 \mathrm{mmol})$, toluene $(1 \mathrm{ml})$ and fitted with an aluminous headspace cap. The reaction is performed under open air at $40 \mathrm{C}$ for $18-24 \mathrm{~h}$ (magnetic stirring bar at $400 \mathrm{rpm}$ ). The crude mixture is directly engaged in silica gel chromatography (usually with n-pentane/ethyl acetate 9:1) for further purification unless stated otherwise. The product spot is usually lower in Rf value than both the starting materials on the TLC plate in the given solvent systems. 


\section{Product characterization and analytic data}<smiles>c1ccc2c(c1)Sc1ccccc1N2c1ccc2c(c1)CCCN2</smiles>

3aa Following the general procedure, the above product was obtained by the cross coupling of phenothiazine and 1,2,3,4-tetrahydroquinoline in 18h. The crude mixture was purified by flash chromatography using n-pentane/ethyl acetate (9:1). Isolated yield: 78\% (dark blue solid). Weight obtained: $129 \mathrm{mg}$

${ }^{1} \mathrm{H}$ NMR $\left(600 \mathrm{MHz}, \mathrm{CDCl}_{3}\right) \delta 7.00-6.91(\mathrm{~m}, 4 \mathrm{H}), 6.86-6.81(\mathrm{~m}, 2 \mathrm{H}), 6.76(\mathrm{t}, J=7.1 \mathrm{~Hz}, 2 \mathrm{H}), 6.67-$ $6.62(\mathrm{~m}, 1 \mathrm{H}), 6.31(\mathrm{~d}, J=8.2 \mathrm{~Hz}, 2 \mathrm{H}), 4.41-3.88(\mathrm{~m}, 1 \mathrm{H}), 3.40-3.36(\mathrm{t}, J=5.4 \mathrm{~Hz}, 2 \mathrm{H}), 2.80(\mathrm{t}, J=6.3$ $\mathrm{Hz}, 2 \mathrm{H}), 2.02-1.96(\mathrm{~m}, 2 \mathrm{H})$.

${ }^{13} \mathrm{C}$ NMR $\left(151 \mathrm{MHz}, \mathrm{CDCl}_{3}\right) \delta 144.9,144.2,131.5,129.5,129.2,126.7,126.4,123.3,121.9,119.4$, $115.7,115.4,41.9,26.9,21.7$.

IR (neat, $\mathrm{cm}^{-1}$ ): 3404, 3058, 2923, 2851, 1599, 1504, 1454, 1296, 1237, 1124, 1037, 922, 815, 740

HRMS (ESI) m/z: [M + H]+ Calculated for $\mathrm{C}_{21} \mathrm{H}_{19} \mathrm{~N}_{2} \mathrm{~S}: 331.12635$, Found: 331.12582<smiles>FC(F)(F)c1ccc2c(c1)N(c1ccc3c(c1)CCCN3)c1ccccc1S2</smiles>

3ba Following the general procedure, the above product was obtained by the cross coupling of 2(trifluoromethyl)-10H-phenothiazine and 1,2,3,4-tetrahydroquinoline in $18 \mathrm{~h}$. The crude mixture was purified by flash chromatography using $n$-pentane/toluene (7:3). Isolated yield: $81 \%$ (pale yellow). Weight obtained: $161 \mathrm{mg}$.

Scale-up of 3ba: Similarly to the general procedure, the above product was obtained by the cross coupling of 2-(trifluoromethyl)-10H- phenothiazine $(3 \mathrm{mmol})$ and 1,2,3,4-tetrahydroquinoline (3 $\mathrm{mmol})$ ) in the presence of silver oxide $(3 \mathrm{mmol})$, in $6 \mathrm{~mL}$ toluene at $50^{\circ} \mathrm{C}$ for $24 \mathrm{~h}$. The crude mixture was purified by flash chromatography using n-pentane/toluene (7:3). Isolated yield: $61 \%$ (pale yellow). Weight obtained: $725 \mathrm{mg}$.

${ }^{1} \mathrm{H}$ NMR $\left(600 \mathrm{MHz}, \mathrm{CDCl}_{3}\right) \delta 7.05-6.97(\mathrm{~m}, 2 \mathrm{H}), 6.96-6.90(\mathrm{~m}, 3 \mathrm{H}), 6.88-6.84(\mathrm{~m}, 1 \mathrm{H}), 6.82-6.77$ $(\mathrm{m}, 1 \mathrm{H}), 6.66-6.61(\mathrm{~m}, 1 \mathrm{H}), 6.52(\mathrm{~s}, 1 \mathrm{H}), 6.34-6.24(\mathrm{~m}, 1 \mathrm{H}), 4.05(\mathrm{~s}, 1 \mathrm{H}), 3.39(\mathrm{t}, J=5.5 \mathrm{~Hz}, 2 \mathrm{H})$, $2.81(\mathrm{t}, J=6.3 \mathrm{~Hz}, 2 \mathrm{H}), 2.06-1.90(\mathrm{~m}, 2 \mathrm{H})$.

${ }^{13} \mathrm{C}$ NMR $\left(151 \mathrm{MHz}, \mathrm{CDCl}_{3}\right.$ ) $\delta 145.4,144.8,144.3,131.1,129.0$ (q, $\left.J=32.76 \mathrm{~Hz}\right), 128.9,128.3,127.21$, $126.5,126.4,124.2,123.5,123.9$ ( $q, J=272.4 \mathrm{~Hz}), 122.6,118.4(q, J=2.1 \mathrm{~Hz}), 116.1,115.5,111.7$ (q, $J=3.9 \mathrm{~Hz}), 41.8,26.9,21.6$. 
${ }^{19}$ F NMR $\left(564 \mathrm{MHz}, \mathrm{CDCl}_{3}\right) \delta-62.7$.

IR (neat, $\mathrm{cm}^{-1}$ ): 3417, 2937, 2840, 1605, 1509, 1467, 1407, 1326, 1247, 1160, 1117, 955, 872, 813, 743

HRMS (ESI) m/z: [M + H]+ Calculated for $\mathrm{C}_{22} \mathrm{H}_{18} \mathrm{~N}_{2} \mathrm{SF}_{3}: 399.11373$, Found: 399.11371<smiles>CC(=O)c1ccc2c(c1)N(c1ccc3c(c1)CCCN3)c1ccccc1S2</smiles>

3ca Following the general procedure, the above product was obtained by the cross coupling of 2(acetyl)-10H-phenothiazine and 1,2,3,4-tetrahydroquinoline in 18h. The crude mixture was purified by flash chromatography using n-pentane/ethyl acetate (7:3). Isolated yield: $74 \%$ (bright yellow solid). Weight obtained: $138 \mathrm{mg}$

${ }^{1} \mathrm{H}$ NMR $\left(600 \mathrm{MHz}, \mathrm{CDCl}_{3}\right) \delta 7.30(\mathrm{dd}, J=7.9,1.6 \mathrm{~Hz}, 1 \mathrm{H}), 6.97(\mathrm{~d}, J=7.9 \mathrm{~Hz}, 1 \mathrm{H}), 6.94-6.89(\mathrm{~m}, 3 \mathrm{H})$, $6.87(\mathrm{~d}, J=1.6 \mathrm{~Hz}, 1 \mathrm{H}), 6.85-6.81(\mathrm{~m}, 1 \mathrm{H}), 6.76(\mathrm{td}, J=7.4,0.8 \mathrm{~Hz}, 1 \mathrm{H}), 6.66-6.59(\mathrm{~m}, 1 \mathrm{H}), 6.27(\mathrm{~d}$, $J=8.2 \mathrm{~Hz}, 1 \mathrm{H}), 4.09(\mathrm{~s}, 1 \mathrm{H}), 3.43-3.29(\mathrm{~m}, 2 \mathrm{H}), 2.79(\mathrm{t}, J=6.3 \mathrm{~Hz}, 2 \mathrm{H}), 2.36(\mathrm{~d}, J=7.8 \mathrm{~Hz}, 3 \mathrm{H}), 1.98$ (dd, $J=11.4,5.9 \mathrm{~Hz}, 2 \mathrm{H}$ ).

${ }^{13} \mathrm{C}$ NMR $\left(151 \mathrm{MHz}, \mathrm{CDCl}_{3}\right) \delta 197.3,145.0,144.7,144.4,135.9,131.0,129.0,128.5,127.2,126.6$, $126.3,126.1,123.4,122.3,122.2,118.1,116.1,115.5,114.2,41.8,26.9,26.4,21.6$.

IR (neat, $\mathrm{cm}^{-1}$ ): 3415, 2935, 2836, 1674, 1595, 1509, 1460, 1399, 1352, 1292, 1232, 1140, 1091, 1039, $935,890,810,743$

HRMS (ESI) m/z: [M + H]+ Calculated for $\mathrm{C}_{23} \mathrm{H}_{21} \mathrm{~N}_{2} \mathrm{SO}: 373.13691$, Found: 373.13696<smiles>N#Cc1ccc2c(c1)N(c1ccc3c(c1)CCCN3)c1ccccc1S2</smiles>

3da Following the general procedure, the above product was obtained by the cross coupling of 2(cyano)-10H-phenothiazine and 1,2,3,4-tetrahydroquinoline in 18h. The crude mixture was purified by flash chromatography using $n$-pentane/ethyl acetate (8:2). Isolated yield: $68 \%$ (yellow solid). Weight obtained: $121 \mathrm{mg}$

${ }^{1} \mathrm{H}$ NMR $\left(600 \mathrm{MHz}, \mathrm{CDCl}_{3}\right) \delta 6.97(\mathrm{dd}, J=7.8,1.1 \mathrm{~Hz}, 1 \mathrm{H}), 6.93(\mathrm{~d}, J=7.8 \mathrm{~Hz}, 1 \mathrm{H}), 6.88(\mathrm{~d}, J=7.4 \mathrm{~Hz}$, $1 \mathrm{H}), 6.87-6.81(\mathrm{~m}, 3 \mathrm{H}), 6.78(\mathrm{t}, J=7.3 \mathrm{~Hz}, 1 \mathrm{H}), 6.63(\mathrm{~d}, J=8.2 \mathrm{~Hz}, 1 \mathrm{H}), 6.42(\mathrm{~d}, J=1.0 \mathrm{~Hz}, 1 \mathrm{H}), 6.28$ $(\mathrm{d}, J=8.2 \mathrm{~Hz}, 1 \mathrm{H}), 4.13(\mathrm{~d}, J=6.9 \mathrm{~Hz}, 1 \mathrm{H}), 3.39(\mathrm{t}, J=5.4 \mathrm{~Hz}, 2 \mathrm{H}), 2.81(\mathrm{t}, J=6.2 \mathrm{~Hz}, 2 \mathrm{H}), 2.03-1.97$ $(m, 2 H)$. 
${ }^{13} \mathrm{C}$ NMR $\left(151 \mathrm{MHz}, \mathrm{CDCl}_{3}\right) \delta 145.4,145.0,143.6,130.9,128.6,127.7,127.4,126.6,126.3,125.2$, $123.5,122.8,119.2,117.7,116.2,116.1,115.7,115.6,109.9,41.8,27.0,21.5$.

IR (neat, $\mathrm{cm}^{-1}$ ): 3404, 2935, 2837, 2224, 2103, 1605, 1508, 1459, 1395, 1296, 1250, 1185, 1129, 1085, $1041,988,938,866,812,744$

HRMS (ESI) m/z: [M + Na]+ Calculated for $\mathrm{C}_{22} \mathrm{H}_{17} \mathrm{~N}_{3} \mathrm{SNa}: 356.12169$, Found: 356.12180<smiles>Clc1ccc2c(c1)N(c1ccc3c(c1)CCCN3)c1ccccc1S2</smiles>

3ea Following the general procedure, the above product was obtained by the cross coupling of 2(chloro)-10H- phenothiazine and 1,2,3,4-tetrahydroquinoline in 18h. The crude mixture was purified by flash chromatography using $n$-pentane/ethyl acetate (20:1). Isolated yield: $54 \%$ (yellow solid). Weight obtained: $98.1 \mathrm{mg}$

${ }^{1} \mathbf{H}$ NMR $\left(600 \mathrm{MHz}, \mathrm{CDCl}_{3}\right) \delta 6.94(\mathrm{dd}, J=7.5 \mathrm{~Hz}, 1.4 \mathrm{~Hz}, 1 \mathrm{H}), 6.92-6.88(\mathrm{~m}, 2 \mathrm{H}), 6.87-6.81(\mathrm{~m}, 2 \mathrm{H})$, $6.79-6.75(\mathrm{td}, J=7.4 \mathrm{~Hz}, 0.8 \mathrm{~Hz}, 1 \mathrm{H}), 6.74-6.70(\mathrm{dd}, J=8.2 \mathrm{~Hz}, 2.0 \mathrm{~Hz}, 1 \mathrm{H}), 6.66-6.62(\mathrm{~m}, 1 \mathrm{H})$, $6.31-6.25(\mathrm{~m}, 2 \mathrm{H}), 4.47-3.71(\mathrm{~m}, 1 \mathrm{H}), 3.39(\mathrm{t}, J=5.5 \mathrm{~Hz}, 2 \mathrm{H}), 2.81(\mathrm{t}, J=6.4 \mathrm{~Hz}, 2 \mathrm{H}), 2.00(\mathrm{~m}, 2 \mathrm{H})$.

${ }^{13} \mathrm{C}$ NMR $\left(151 \mathrm{MHz}, \mathrm{CDCl}_{3}\right) \delta 146.1,144.6,144.3,132.5,131.2,129.0,128.7,127.0,126.9,126.4$, $123.4,122.4,121.6,119.1,117.8,116.0,115.6,115.5,41.8,26.9,21.6$.

IR (neat, $\mathrm{cm}^{-1}$ ): 3406, 2924, 2838, 2320, 2091, 1874, 1788, 1563, 1505, 1455, 1390, 1286, 1236, 1126, 1094, 1039, 939, 859, 813, 745.

HRMS (ESI) m/z: [M + Na]+ Calculated for $\mathrm{C}_{21} \mathrm{H}_{17} \mathrm{~N}_{2} \mathrm{SCINa:} 387.06932$, Found: 387.06940<smiles>CS(=O)(=O)c1ccc2c(c1)N(c1ccc3c(c1)CCCN3)c1ccccc1S2</smiles>

3fa Following the general procedure, the above product was obtained by the cross coupling of 2(methylsulfonyl)-10H- phenothiazine and 1,2,3,4-tetrahydroquinoline in 18h. The crude mixture was purified by flash chromatography using $n$-pentane/ethyl acetate $(9: 1)$. Isolated yield: $57 \%$ (pale yellow solid). Weight obtained: $116 \mathrm{mg}$

${ }^{1}$ H NMR $\left(600 \mathrm{MHz}, \mathrm{CDCl}_{3}\right) \delta 7.28-7.24(\mathrm{~m}, 1 \mathrm{H}), 7.05(\mathrm{~d}, J=7.9 \mathrm{~Hz}, 1 \mathrm{H}), 6.93-6.88(\mathrm{~m}, 3 \mathrm{H}), 6.87-$ $6.83(\mathrm{~m}, 1 \mathrm{H}), 6.82-6.76(\mathrm{~m}, 2 \mathrm{H}), 6.67-6.61(\mathrm{~m}, 1 \mathrm{H}), 6.25(\mathrm{dd}, J=8.2 \mathrm{~Hz}, 1.1 \mathrm{~Hz}, 1 \mathrm{H}), 4.39-3.87(\mathrm{~m}$, $1 \mathrm{H}), 3.39(\mathrm{t}, \mathrm{J}=5.5 \mathrm{~Hz}, 2 \mathrm{H}), 2.89(\mathrm{~s}, 3 \mathrm{H}), 2.79(\mathrm{t}, \mathrm{J}=6.3 \mathrm{~Hz}, 2 \mathrm{H}), 1.99(\mathrm{~m}, 2 \mathrm{H})$.

${ }^{13} \mathrm{C}$ NMR $\left(151 \mathrm{MHz}, \mathrm{CDCl}_{3}\right) \delta 145.8,144.9,144.0,138.8,130.8,128.6,127.9,127.4,127.2,126.7$, $126.4,123.6,122.8,120.4,117.9,116.4,115.7,112.9,44.2,44.7,26.9,21.5$. 
IR (neat, $\mathrm{cm}^{-1}$ ): 3749, 3407, 3065, 3022, 2926, 2843, 2584, 2342, 2190, 2110, 1986, 1879, 1736, 1676, $1607,1510,1457,1390,1290,1238,1144,1095,953,906,875,816,783,738$.

HRMS (ESI) m/z: [M + H]+ Calculated for $\mathrm{C}_{22} \mathrm{H}_{21} \mathrm{O}_{2} \mathrm{~N}_{2} \mathrm{~S}_{2}: 409.10390$, Found: 409.10379<smiles>c1ccc2c(c1)Oc1ccccc1N2c1ccc2c(c1)CCCN2</smiles>

3ga Following the general procedure, the above product was obtained by the cross coupling of phenoxazine and 1,2,3,4-tetrahydroquinoline in 18h. The crude mixture was purified by flash chromatography using n-pentane/ethyl acetate (9:1). Isolated yield: $47 \%$ (yellow solid). Weight obtained: $74 \mathrm{mg}$

${ }^{1} \mathbf{H}$ NMR $\left(600 \mathrm{MHz}, \mathrm{CDCl}_{3}\right) \delta 6.88-6.84(\mathrm{~m}, 2 \mathrm{H}), 6.70-6.54(\mathrm{~m}, 7 \mathrm{H}), 6.05-5.99(\mathrm{~m}, 2 \mathrm{H}), 4.51-3.67$ $(\mathrm{m}, 1 \mathrm{H}), 3.37(\mathrm{t}, J=5.6 \mathrm{~Hz}, 2 \mathrm{H}), 2.78(\mathrm{t}, J=6.4 \mathrm{~Hz}, 2 \mathrm{H}), 1.98(\mathrm{~m}, 2 \mathrm{H}) \mathrm{ppm}$.

${ }^{13} \mathrm{C}$ NMR $\left(151 \mathrm{MHz}, \mathrm{CDCl}_{3}\right) \delta 144.4,144.0,135.1,131.1,128.6,127.3,123.5,123.1,120.7,115.7$, 115.0, 113.3, 41.9, 26.9, 21.7.

IR (neat, cm ${ }^{-1}$ ): 3403, 3057, 2925, 2841, 2079, 1877, 1737, 1591, 1479, 1329, 1264, 1196, 1113, 1039, $963,921,852,810,736$.

HRMS (ESI) $m / z:[M+H]+$ Calculated for $\mathrm{C}_{21} \mathrm{H}_{19} \mathrm{~N}_{2} \mathrm{O}: 315.14919$, Found: 315.14844<smiles>CC1CCNc2ccc(N3c4ccccc4Sc4ccc(C(F)(F)F)cc43)cc21</smiles>

3bb Following the general procedure, the above product was obtained by the cross coupling of 2(trifluoromethyl)-10H- phenothiazine $(0.2 \mathrm{mmol})$ and 4-methyl-1,2,3,4-tetrahydroquinoline $(0.2$ mmol) using silver oxide $(0.2 \mathrm{mmol})$ and toluene $(0.5 \mathrm{ml})$ in $18 \mathrm{~h}$. The crude mixture was purified by flash chromatography using n-pentane/ethyl acetate (9:1). Isolated yield: 68\% (pale yellow oil). Weight obtained: $56 \mathrm{mg}$

${ }^{1} \mathrm{H}$ NMR $\left(600 \mathrm{MHz}, \mathrm{CDCl}_{3}\right) \delta 7.03-6.99(\mathrm{~m}, 2 \mathrm{H}), 6.98(\mathrm{dd}, J=7.9,0.7 \mathrm{~Hz}, 1 \mathrm{H}), 6.95-6.89(\mathrm{~m}, 2 \mathrm{H})$, $6.87-6.82(\mathrm{~m}, 1 \mathrm{H}), 6.79(\mathrm{td}, J=7.4,1.2 \mathrm{~Hz}, 1 \mathrm{H}), 6.65(\mathrm{~d}, J=8.3 \mathrm{~Hz}, 1 \mathrm{H}), 6.48(\mathrm{~d}, J=1.0 \mathrm{~Hz}, 1 \mathrm{H}), 6.27$ $(\mathrm{dd}, J=8.2,1.1 \mathrm{~Hz}, 1 \mathrm{H}), 4.51-3.90(\mathrm{~m}, 1 \mathrm{H}), 3.45-3.34(\mathrm{~m}, 2 \mathrm{H}), 2.99-2.90(\mathrm{~m}, 1 \mathrm{H}), 2.08-1.99(\mathrm{~m}$, $1 \mathrm{H}), 1.78-1.69(\mathrm{~m}, 1 \mathrm{H}), 1.29(\mathrm{~d}, J=7.0 \mathrm{~Hz}, 3 \mathrm{H})$.

${ }^{13} \mathrm{C}$ NMR $\left(151 \mathrm{MHz}, \mathrm{CDCl}_{3}\right) \delta 145.4,144.4,144.1,130.1,129.0$ (q, $\left.J=32.1 \mathrm{~Hz}\right), 128.8,128.7,128.5$, $127.1,126.4,126.4,124.1,123.9$ ( $q, J=272.5 \mathrm{~Hz}$ ), 122.5, 118.4 (q, $J=3.9 \mathrm{~Hz}$ ), 116.1, 115.6, 111.7 (q, $J=3.9 \mathrm{~Hz}), 39.0,30.3,29.3,22.3$. 
${ }^{19}$ F NMR (376 MHz, dmso) $\delta-61.78$.

IR (neat, $\mathrm{cm}^{-1}$ ): 3419, 2926, 2854, 1607, 1570, 1508, 1466, 1441, 1408, 1326, 1247, 1163, 1119, 1088, $1043,959,907,873,814,742$

HRMS (ESI) m/z: [M + H] + Calculated for $\mathrm{C}_{23} \mathrm{H}_{20} \mathrm{~N}_{2} \mathrm{SF}_{3}: 413.12938$, Found: 413.12927<smiles>CC(=O)c1ccc2c(c1)N(c1ccc3c(c1)C(C)CCN3)c1ccccc1S2</smiles>

3cb Following the general procedure, the above product was obtained by the cross coupling of 2(acetyl)-10H-phenothiazine and 4-methyl-1,2,3,4-tetrahydroquinoline in $24 \mathrm{~h}$. The crude mixture was purified by flash chromatography using n-pentane/ethyl acetate (10:1). The product was obtained as a mixture of diastereomers. Isolated yield: 75\% (dark yellow oil). Weight obtained: $145 \mathrm{mg}$

${ }^{1}$ H NMR $\left(600 \mathrm{MHz}, \mathrm{CDCl}_{3}\right) \delta 7.32(\mathrm{dd}, \mathrm{J}=7.9 \mathrm{~Hz}, 1.7 \mathrm{~Hz}, 1 \mathrm{H}), 7.02(\mathrm{~d}, \mathrm{~J}=1.7 \mathrm{~Hz}, 1 \mathrm{H}), 6.98(\mathrm{~d}, \mathrm{~J}=7.9$ $\mathrm{Hz}, 1 \mathrm{H}), 6.93-6.91(\mathrm{~m}, 2 \mathrm{H}), 6.87(\mathrm{~d}, \mathrm{~J}=1.7 \mathrm{~Hz}, 1 \mathrm{H}), 6.84(\mathrm{td}, \mathrm{J}=7.8 \mathrm{~Hz}, 1.5 \mathrm{~Hz}, 1 \mathrm{H}), 6.76(\mathrm{td}, \mathrm{J}=7.4$ $\mathrm{Hz}, 1.1 \mathrm{~Hz}, 1 \mathrm{H}), 6.64(\mathrm{~d}, \mathrm{~J}=8.3 \mathrm{~Hz}, 1 \mathrm{H}), 6.26(\mathrm{dd}, \mathrm{J}=8.3 \mathrm{~Hz}, 1.0 \mathrm{~Hz}, 1 \mathrm{H}), 4.28-4.01(\mathrm{~m}, 1 \mathrm{H}), 3.42-$ $3.33(\mathrm{~m}, 2 \mathrm{H}), 2.98-2.92(\mathrm{~m}, 1 \mathrm{H}), 2.36(\mathrm{~s}, 3 \mathrm{H}), 2.05-2.00(\mathrm{~m}, 1 \mathrm{H}), 1.75-1.70(\mathrm{~m}, 1 \mathrm{H}), 1.30(\mathrm{~d}, \mathrm{~J}=7.0$ $\mathrm{Hz}, 3 \mathrm{H})$,

${ }^{13} \mathrm{C}$ NMR $\left(151 \mathrm{MHz}, \mathrm{CDCl}_{3}\right) \delta 197.2,145.1,144.5,144.2,135.9,130.2,128.8,128.6,128.6,127.2$, $126.5,126.3,126.1,122.3,122.2,118.2,116.1,115.7,114.4,39.0,30.3,29.3,26.3,22.5$.

IR (neat, $\mathrm{cm}^{-1}$ ): 3845, 3637, 3397, 2924, 2854, 2690, 2471, 2311, 2114, 1976, 1894, 1750, 1673, 1594, 1507, 1461, 1398, 1355, 1291, 1234, 1130, 1096, 1043, 934, 883, 811, 738.

HRMS (ESI) m/z: [M + H]+ Calculated for $\mathrm{C}_{24} \mathrm{H}_{23} \mathrm{~N}_{2} \mathrm{OS}: 387.15256$, Found: 387.15234<smiles>CC1CCNc2ccc(N3c4ccccc4Sc4ccc(C#N)cc43)cc21</smiles>

3db Following the general procedure, the above product was obtained by the cross coupling of 2(cyano)-10H-phenothiazine and 4-methyl-1,2,3,4-tetrahydroquinoline in $24 \mathrm{~h}$. The crude mixture was purified by flash chromatography using n-pentane/ethyl acetate (4:1). Isolated yield: $67 \%$ (yellow oil). Weight obtained: $124 \mathrm{mg}$

${ }^{1} \mathrm{H}$ NMR $\left(600 \mathrm{MHz}, \mathrm{CDCl}_{3}\right) \delta 6.98-6.93(\mathrm{~m}, 3 \mathrm{H}), 6.90(\mathrm{dd}, J=7.5 \mathrm{~Hz}, 1.56 \mathrm{~Hz}, 1 \mathrm{H}), 6.87-6.83(\mathrm{~m}$, $2 \mathrm{H}), 6.80-6.77(\mathrm{td}, J=7.4 \mathrm{~Hz}, 1.1 \mathrm{~Hz}, 1 \mathrm{H}), 6.64(\mathrm{~d}, J=8.3 \mathrm{~Hz}, 1 \mathrm{H}), 6.40(\mathrm{~d}, J=1.5 \mathrm{~Hz}, 1 \mathrm{H}), 6.26(\mathrm{dd}$, 
$8.3 \mathrm{~Hz}, 1.1 \mathrm{~Hz}, 1 \mathrm{H}), 4.44-3.90(\mathrm{~m}, 1 \mathrm{H}), 3.44-3.36(\mathrm{~m}, 2 \mathrm{H}), 2.98-2.93(\mathrm{~m}, 1 \mathrm{H}), 2.06-2.01(\mathrm{~m}, 1 \mathrm{H})$, $1.76-1.71(\mathrm{~m}, 1 \mathrm{H}), 1.30(\mathrm{~d}, J=7.0 \mathrm{~Hz}, 3 \mathrm{H})$.

${ }^{13} \mathrm{C}$ NMR $\left(151 \mathrm{MHz}, \mathrm{CDCl}_{3}\right) \delta 145.5,144.5,143.8,129.7,128.9,128.6,127.4,126.6,126.5,125.2$, $126.4,125.2,122.8,119.2,117.8,117.7,116.1,115.7,110.0,39.0,30.3,29.3,22.3$.

IR (neat, $\mathrm{cm}^{-1}$ ): 3406, 3062, 2925, 2853, 2581, 2331, 2226, 1877, 1731, 1607, 1552, 1508, 1460, 1397, $1354,1295,1242,1195,1130,1045,987,907,865,812,732$.

HRMS (ESI) m/z: $[\mathrm{M}+\mathrm{H}]+$ Calculated for $\mathrm{C}_{23} \mathrm{H}_{20} \mathrm{~N}_{3} \mathrm{~S}: 370.13718$, Found: 370.13724<smiles>CC1CCNc2ccc(N3c4ccccc4Sc4ccc(Cl)cc43)cc21</smiles>

3eb Following the general procedure, the above product was obtained by the cross coupling of 2(chloro)-10H- phenothiazine and 4-methyl-1,2,3,4-tetrahydroquinoline in $24 \mathrm{~h}$. The crude mixture was purified by flash chromatography using n-pentane/ethyl acetate (10:1). Isolated yield: $34 \%$ (white solid). Weight obtained: $65 \mathrm{mg}$

${ }^{1} \mathrm{H}$ NMR $\left(600 \mathrm{MHz}, \mathrm{CDCl}_{3}\right) \delta 7.02(\mathrm{~d}, \mathrm{~J}=1.7 \mathrm{~Hz}, 1 \mathrm{H}), 6.95$ (dd, J = 7.5 Hz, $\left.1.4 \mathrm{~Hz}, 1 \mathrm{H}\right), 6.92$ (dd, $8.3 \mathrm{~Hz}$, $2.2 \mathrm{~Hz}, 1 \mathrm{H}), 6.87-6.83(\mathrm{~m}, 2 \mathrm{H}), 6.78(\mathrm{t}, 7.4 \mathrm{~Hz}, 1 \mathrm{H}), 6.73(\mathrm{dd}, \mathrm{J}=8.2 \mathrm{~Hz}, 2.0 \mathrm{~Hz}, 1 \mathrm{H}), 6.69(\mathrm{~d}, \mathrm{~J}=8.3$ $\mathrm{Hz}, 1 \mathrm{H}), 6.28-6.27(\mathrm{~m}, 2 \mathrm{H}), 4.55-3.85(\mathrm{~m}, 1 \mathrm{H}), 3.45-3.36(\mathrm{~m}, 2 \mathrm{H}), 2.99-2.93(\mathrm{~m}, 1 \mathrm{H}), 2.08-2.03$ $(m, 1 H), 1.77-1.72(m, 1 H), 1.30(d, J=7.0 \mathrm{~Hz}, 3 \mathrm{H})$.

${ }^{13} \mathrm{C}$ NMR $\left(151 \mathrm{MHz}, \mathrm{CDCl}_{3}\right) \delta 146.1,144.4,143.5,132.6,130.2,129.2,129.1,128.9,127.0,126.9$, $126.4,122.4,121.7,119.1,117.9,116.0,115.9,115.7,39.1,30.2,29.3,22.3$.

IR (neat, $\mathrm{cm}^{-1}$ ): 3417, 3061, 2930, 2853, 2570, 2334, 2243, 2086, 1860, 1779, 1567, 1505, 1453, 1390, $1288,1242,1100,1044,951,907,853,802,732$.

HRMS (ESI) m/z: [M + H]+ Calculated for $\mathrm{C}_{22} \mathrm{H}_{20} \mathrm{CIN}_{2} \mathrm{~S}: 379.10302$, Found: 379.10248<smiles>CC1Cc2cc(N3c4ccccc4Sc4ccc(C(F)(F)F)cc43)ccc2N1</smiles>

3bc Following the general procedure, the above product was obtained by the cross coupling of 2(trifluoromethyl)-10H- phenothiazine $(0.5 \mathrm{mmol})$ and 4-methyl-1,2,3,4-tetrahydroquinoline $(0.5$ mmol) using silver oxide $(0.5 \mathrm{mmol})$ and toluene $(1 \mathrm{ml})$ in $18 \mathrm{~h}$. The crude mixture was purified by flash chromatography using n-pentane/ethyl acetate (9:1). Isolated yield: 70\% (pale yellow oil). Weight obtained: $140 \mathrm{mg}$ 
${ }^{1} \mathrm{H}$ NMR $(600 \mathrm{MHz}, \mathrm{dmso}) \delta 7.16(\mathrm{~d}, J=7.9 \mathrm{~Hz}, 1 \mathrm{H}), 7.07(\mathrm{~d}, J=7.9 \mathrm{~Hz}, 1 \mathrm{H}), 7.00-6.95(\mathrm{~m}, 2 \mathrm{H}), 6.91$ $(\mathrm{m}, 2 \mathrm{H}), 6.81(\mathrm{td}, J=7.4,0.7 \mathrm{~Hz}, 1 \mathrm{H}), 6.66(\mathrm{~d}, J=8.4 \mathrm{~Hz}, 1 \mathrm{H}), 6.34(\mathrm{~d}, J=1.2 \mathrm{~Hz}, 1 \mathrm{H}), 6.21(\mathrm{dd}, J=8.4$, $0.6 \mathrm{~Hz}, 1 \mathrm{H}), 6.01(\mathrm{~s}, 1 \mathrm{H}), 4.01-3.95(\mathrm{~m}, 1 \mathrm{H}), 3.12(\mathrm{dd}, J=16.2,9.0 \mathrm{~Hz}, 1 \mathrm{H}), 2.56(\mathrm{dd}, J=16.2,8.4 \mathrm{~Hz}$, $1 \mathrm{H}), 1.22(\mathrm{~d}, J=6.0 \mathrm{~Hz}, 3 \mathrm{H})$.

${ }^{13}$ C NMR (151 MHz, dmso) $\delta$ 152.5, 145.4, 143.9, 136.7, 131.6, 129.5, 128.0 (q, J = $32.1 \mathrm{~Hz}$ ), 127.3, $126.8,126.3,124.3(q, J=272.2 \mathrm{~Hz}), 123.3,118.8(q, J=3.8 \mathrm{~Hz}), 117.8,116.4,115.4,111.2$ (q, $J=2.8$ $\mathrm{Hz}), 109.4,55.3,37.5,22.4$.

${ }^{19}$ F NMR (564 MHz, dmso) $\delta-61.66$.

IR (neat, $\mathrm{cm}^{-1}$ ): 3384, 2961, 2925, 2854, 1606, 1488, 1468, 1439, 1407, 1326, 1243, 1164, 1117, 1087, $1043,957,872,814,743$

HRMS (ESI) m/z: [M + H] + Calculated for $\mathrm{C}_{22} \mathrm{H}_{18} \mathrm{~N}_{2} \mathrm{SF}_{3}: 399.11373$, Found: 399.11383<smiles>CC(=O)c1ccc2c(c1)N(c1ccc3c(c1)CC(C)N3)c1ccccc1S2</smiles>

3cc Following the general procedure, the above product was obtained by the cross coupling of 2(acetyl)-10H- phenothiazine and 2-methylindoline in $24 \mathrm{~h}$. The crude mixture was purified by flash chromatography using n-pentane/ethyl acetate (6:4). Isolated yield: 61\% (bright yellow oil). Weight obtained: $113.2 \mathrm{mg}$

${ }^{1} \mathrm{H}$ NMR $(600 \mathrm{MHz}, \mathrm{dmso}) \delta 7.39$ (dd, $\left.J=7.9,1.7 \mathrm{~Hz}, 1 \mathrm{H}\right), 7.09$ (d, $\left.J=7.9 \mathrm{~Hz}, 1 \mathrm{H}\right), 7.00-6.93(\mathrm{~m}, 2 \mathrm{H})$, $6.92-6.87(\mathrm{~m}, 2 \mathrm{H}), 6.79(\mathrm{td}, J=7.5,1.0 \mathrm{~Hz}, 1 \mathrm{H}), 6.71(\mathrm{~d}, J=1.7 \mathrm{~Hz}, 1 \mathrm{H}), 6.66(\mathrm{~d}, J=8.1 \mathrm{~Hz}, 1 \mathrm{H}), 6.18$ (dd, $J=8.3,0.8 \mathrm{~Hz}, 1 \mathrm{H}), 5.98(\mathrm{~d}, J=1.5 \mathrm{~Hz}, 1 \mathrm{H}), 4.03-3.96(\mathrm{~m}, 1 \mathrm{H}), 3.13(\mathrm{dd}, J=15.9,8.7 \mathrm{~Hz}, 1 \mathrm{H})$, $2.57(\mathrm{dd}, J=15.9,8.0 \mathrm{~Hz}, 1 \mathrm{H}), 1.24(\mathrm{~d}, J=6.1 \mathrm{~Hz}, 3 \mathrm{H})$.

${ }^{13}$ C NMR (151 MHz, dmso) $\delta 197.1,152.4,144.8,144.1,136.0,131.5,129.6,128.6,127.9,126.7$, $126.6,126.4,125.8,123.4,122.8,117.6,116.3,113.6,109.4,55.3,37.6,26.7,22.5$.

IR (neat, $\mathrm{cm}^{-1}$ ): 3377, 2962, 1671, 1588, 1487, 1463, 1400, 1356, 1296, 1236, 1129, 1098, 994, 887, 823,748

HRMS (ESI) m/z: [M + H] + Calculated for $\mathrm{C}_{23} \mathrm{H}_{21} \mathrm{~N}_{2} \mathrm{SO}: 373.13691$, Found: 373.13663

HPLC profile of the $3 \mathrm{cc}$ racemate (AD-H, $1 \mathrm{~mL} / \mathrm{min}$, hexane/EtOH: $8 / 2$ ) (top), and HPLC profile of the enantiopure 3cc: 
Data file: PYV-60 ADH 8020 EtOH flow1 20 DATA

Method: HPLC1 ADH 8020 EtOH flow1_acq 45

Date: $27.06 .201 \overline{9} 17 \cdot 5 \overline{6} \cdot 11$
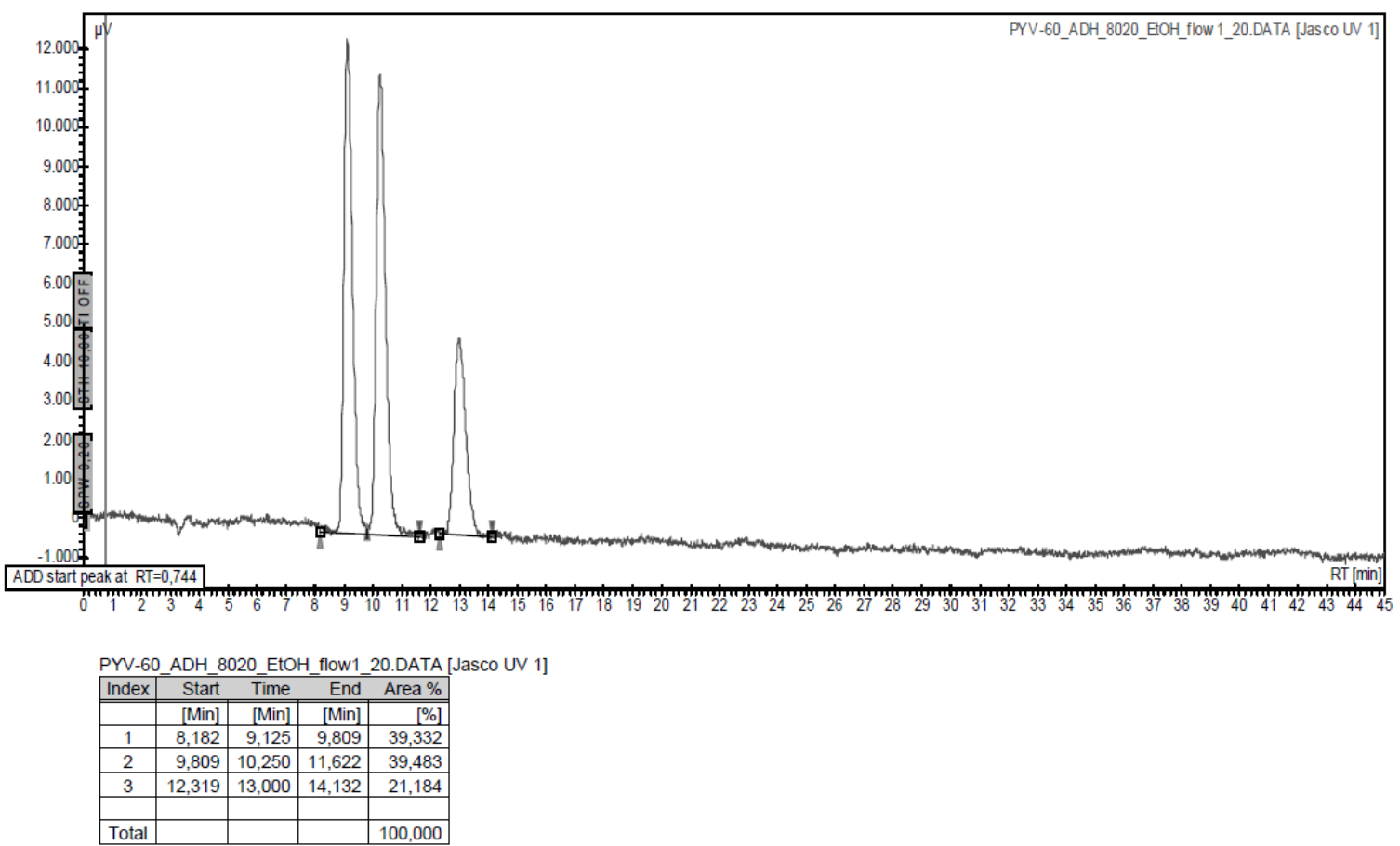

Data file: PYV-64 ADH 8020 EtOH flow1 21.DATA

Method: HPLC1_ADH_-8020_EtOH_flow1_acq_45

Date: $27.06 .201 \overline{9} 18: 4 \overline{3}: 51$

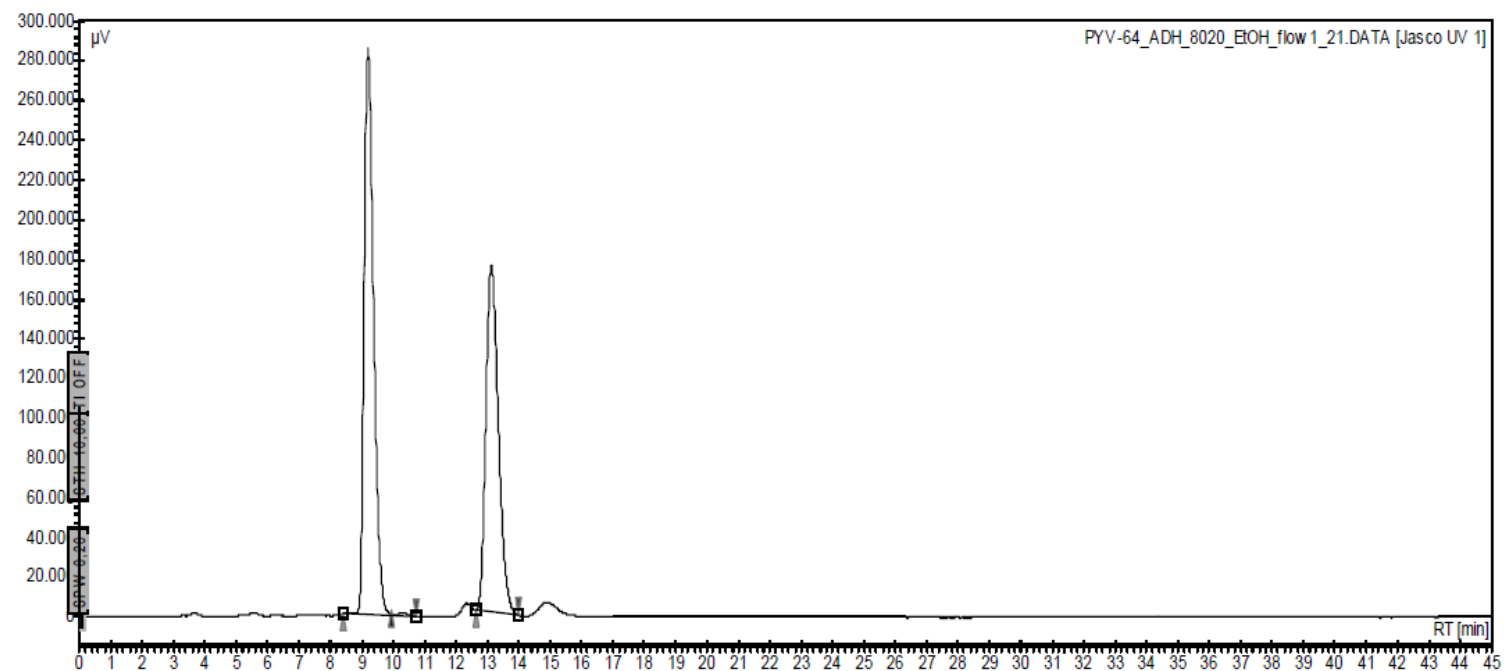

PYV-64_ADH_8020_EtOH_flow1_21.DATA [Jasco UV 1]

\begin{tabular}{|c|c|c|c|c|}
\hline ndex & Start & Time & End & Area \% \\
\hline & [Min] & [Min] & [Min] & [\%] \\
\hline 1 & 8,414 & 9,208 & 9,948 & 55,183 \\
\hline 2 & 9,948 & 10,308 & 10,739 & 0,237 \\
\hline 3 & 12,645 & 13,133 & 13,993 & 44,580 \\
\hline & & & & \\
\hline
\end{tabular}


<smiles>CC1Cc2cc(N3c4ccccc4Sc4ccc(C#N)cc43)ccc2N1</smiles>

3dc Following the general procedure, the above product was obtained by the cross coupling of 2(cyano)- $10 \mathrm{H}$ - phenothiazine and 2-methylindoline in $24 \mathrm{~h}$. The crude mixture was purified by flash chromatography using n-pentane/ethyl acetate (8:2). Isolated yield: $55 \%$ (yellow oil). Weight obtained: $97 \mathrm{mg}$

${ }^{1} \mathrm{H}$ NMR $\left(600 \mathrm{MHz}, \mathrm{CDCl}_{3}\right) \delta 6.99-6.95(\mathrm{~m}, 2 \mathrm{H}), 6.99-6.93(\mathrm{~m}, 3 \mathrm{H}), 6.94(\mathrm{~d}, J=7.9 \mathrm{~Hz}, 1 \mathrm{H}), 6.93-$ $6.88(\mathrm{~m}, 2 \mathrm{H}), 6.86-6.82(\mathrm{~m}, 1 \mathrm{H}), 6.78(\mathrm{td}, J=10.8,4.2 \mathrm{~Hz}, 1 \mathrm{H}), 6.74(\mathrm{~d}, J=8.4 \mathrm{~Hz}, 1 \mathrm{H}), 6.39(\mathrm{~d}, J=$ $1.2 \mathrm{~Hz}, 1 \mathrm{H}), 6.25(\mathrm{dd}, J=8.4,1.2 \mathrm{~Hz}, 1 \mathrm{H}), 4.21-4.11(\mathrm{~m}, 1 \mathrm{H}), 4.05(\mathrm{~s}, 1 \mathrm{H}), 3.24(\mathrm{dd}, J=15.8,8.7 \mathrm{~Hz}$, $1 \mathrm{H}), 2.74(\mathrm{dd}, J=15.8,8.1 \mathrm{~Hz}, 1 \mathrm{H}), 1.38(\mathrm{~d}, J=6.2 \mathrm{~Hz}, 3 \mathrm{H})$.

${ }^{13} \mathrm{C}$ NMR $\left(151 \mathrm{MHz}, \mathrm{CDCl}_{3}\right) \delta 151.3,145.4,143.6,131.9,129.6,129.5,127.3,126.6,126.5,126.3$, $125.2,122.8,119.1,117.7,117.6,116.1,110.2,109.9,55.7,37.6,22.3$.

IR (neat, $\mathrm{cm}^{-1}$ ): 3386, 2964, 2922, 2853, 2226, 1611, 1590, 1553, 1489, 1463, 1399, 1301, 1261, 1102, $1042,939,868,811,740,616$

HRMS (ESI) m/z: [M + H]+ Calculated for $\mathrm{C}_{22} \mathrm{H}_{18} \mathrm{~N}_{3} \mathrm{~S}: 356.12159$, Found: 356.12161<smiles>CC1Cc2cc(N3c4ccccc4Sc4ccc(Cl)cc43)ccc2N1</smiles>

3ec Following the general procedure, the above product was obtained by the cross coupling of 2(chloro)-10H-phenothiazine and 2-methylindoline in $24 \mathrm{~h}$. The crude mixture was purified by flash chromatography using n-pentane/ethyl acetate (10:1). Isolated yield: $58 \%$ (white oil). Weight obtained: $106 \mathrm{mg}$

${ }^{1} \mathrm{H}$ NMR $(600 \mathrm{MHz}, \mathrm{dmso}) \delta 6.99-6.93(\mathrm{~m}, 3 \mathrm{H}), 6.93-6.86(\mathrm{~m}, 2 \mathrm{H}), 6.83-6.77(\mathrm{~m}, 2 \mathrm{H}), 6.63(\mathrm{~d}, J=$ $7.8 \mathrm{~Hz}, 1 \mathrm{H}), 6.20(\mathrm{~d}, J=8.4 \mathrm{~Hz}, 1 \mathrm{H}), 6.11(\mathrm{~d}, J=2.4 \mathrm{~Hz}, 1 \mathrm{H}), 6.00(\mathrm{~s}, 1 \mathrm{H}), 4.03-3.94(\mathrm{~m}, 1 \mathrm{H}), 3.12(\mathrm{dd}$, $J=16.2,9.0 \mathrm{~Hz}, 1 \mathrm{H}), 2.56(\mathrm{dd}, J=16.2,9.0 \mathrm{~Hz}, 1 \mathrm{H}), 1.22(\mathrm{~d}, J=6.0 \mathrm{~Hz}, 3 \mathrm{H})$.

${ }^{13}$ C NMR (151 MHz, dmso) $\delta 152.5,146.2,144.0,131.9,131.6,129.5,128.3,127.9,127.8,126.8$, $126.4,123.1,122.0,118.4,117.9,116.3,115.3,109.3,55.3,37.6,22.5$.

IR (neat, $\mathrm{cm}^{-1}$ ): 3382, 2961, 2923, 1650, 1611, 1587, 1564, 1486, 1456, 1390, 1289, 1239, 1125, 1099, $1024,995,953,884,849,821,798,743$

HRMS (ESI) m/z: [M + H]+ Calculated for $\mathrm{C}_{21} \mathrm{H}_{18} \mathrm{ClN}_{2} \mathrm{~S}$ : 365.08737 ; Found: 365.08704 


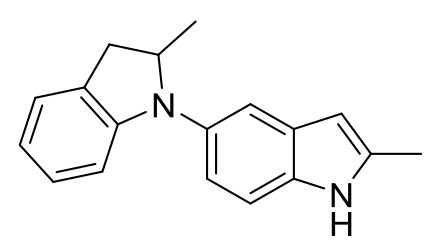

4cc Similar to the general procedure, the above product was obtained by the homo coupling of 2Methyl indoline $(0.5 \mathrm{mmol})$ in the presence of silver oxide $(1 \mathrm{mmol})$ at room temperature for $18 \mathrm{~h}$. The crude mixture was purified by flash chromatography using n-pentane/ethyl acetate (9:1). Isolated yield: $70 \%$ (yellow oil). Weight obtained: $46 \mathrm{mg}$

${ }^{1} \mathrm{H}$ NMR (400 MHz, CDCl $) \delta 7.84(\mathrm{~s}, 1 \mathrm{H}), 7.39(\mathrm{~s}, 1 \mathrm{H}), 7.29(\mathrm{~d}, J=8.4 \mathrm{~Hz}, 1 \mathrm{H}), 7.11(\mathrm{~d}, J=7.2 \mathrm{~Hz}, 1 \mathrm{H})$, $7.03(\mathrm{dd}, J=8.8,2.0 \mathrm{~Hz}, 1 \mathrm{H}), 6.96(\mathrm{t}, J=7.6 \mathrm{~Hz}, 1 \mathrm{H}), 6.66(\mathrm{t}, J=7.2 \mathrm{~Hz}, 1 \mathrm{H}), 6.46(\mathrm{~d}, J=8.0 \mathrm{~Hz}, 1 \mathrm{H})$, $6.20(\mathrm{~d}, J=0.8 \mathrm{~Hz}, 1 \mathrm{H}), 4.31-4.16(\mathrm{~m}, 1 \mathrm{H}), 3.28(\mathrm{dd}, J=15.2,8.4 \mathrm{~Hz}, 1 \mathrm{H}), 2.79(\mathrm{dd}, J=15.3,9.6 \mathrm{~Hz}$, $1 \mathrm{H}), 2.45(\mathrm{~s}, 3 \mathrm{H}), 1.30(\mathrm{~d}, J=6.1 \mathrm{~Hz}, 3 \mathrm{H})$.

${ }^{13} \mathrm{C}$ NMR $\left(101 \mathrm{MHz}, \mathrm{CDCl}_{3}\right) \delta 152.2,136.3,135.8,133.8,129.7,128.9,127.1,124.2,119.4,117.5$, $116.4,110.8,107.7,100.4,62.1,37.3,19.9,13.7$.

IR (neat, $\mathrm{cm}^{-1}$ ): 3399, 3045, 2962, 2923, 1599, 1479, 1408, 1374, 1290, 1258, 1191, 1164, 1092, 1054, $1020,948,903,778,741$

HRMS (ESI) m/z: $[\mathrm{M}+\mathrm{H}]+$ Calculated for $\mathrm{C}_{18} \mathrm{H}_{19} \mathrm{~N}_{2}: 263.15428$, Found: 263.15427 


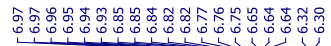

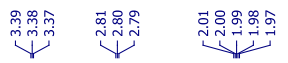<smiles>c1ccc2c(c1)SCCN2c1ccc2sc3ccccc3c2c1</smiles>
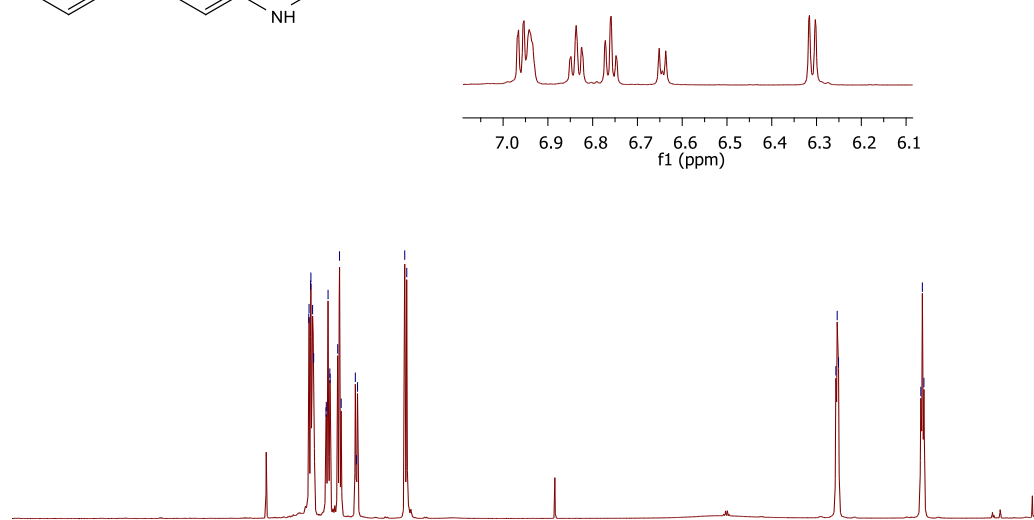

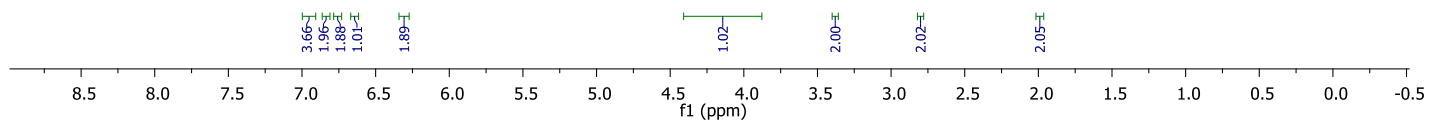

$3 a a$

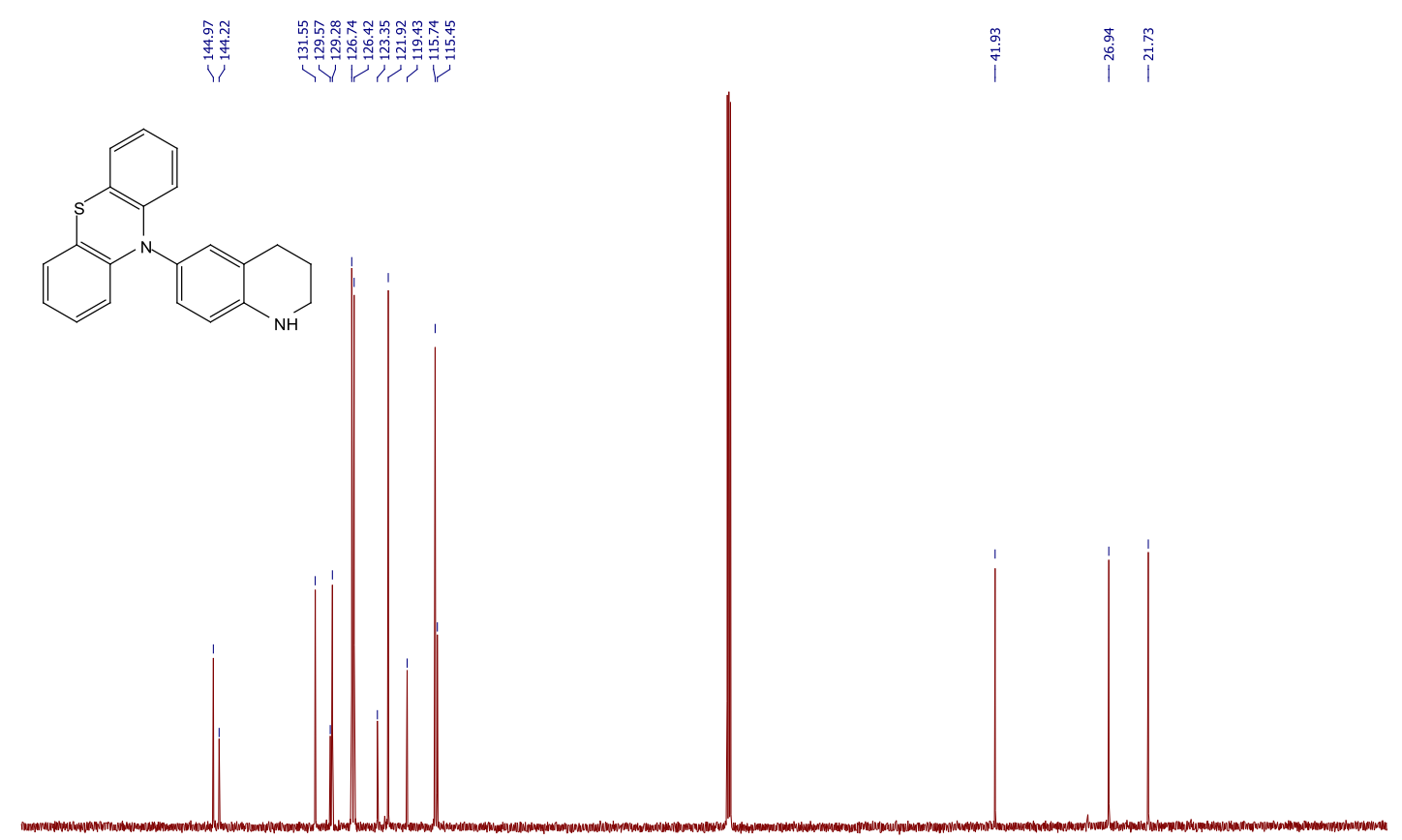

$\begin{array}{rlllllllllllllllll}170 & 160 & 150 & 140 & 130 & 120 & 110 & 100 & 90 & \underset{\mathrm{f} 1(\mathrm{ppm})}{80} & 70 & 60 & 50 & 40 & 30 & 20 & 10 & 0\end{array}$ 


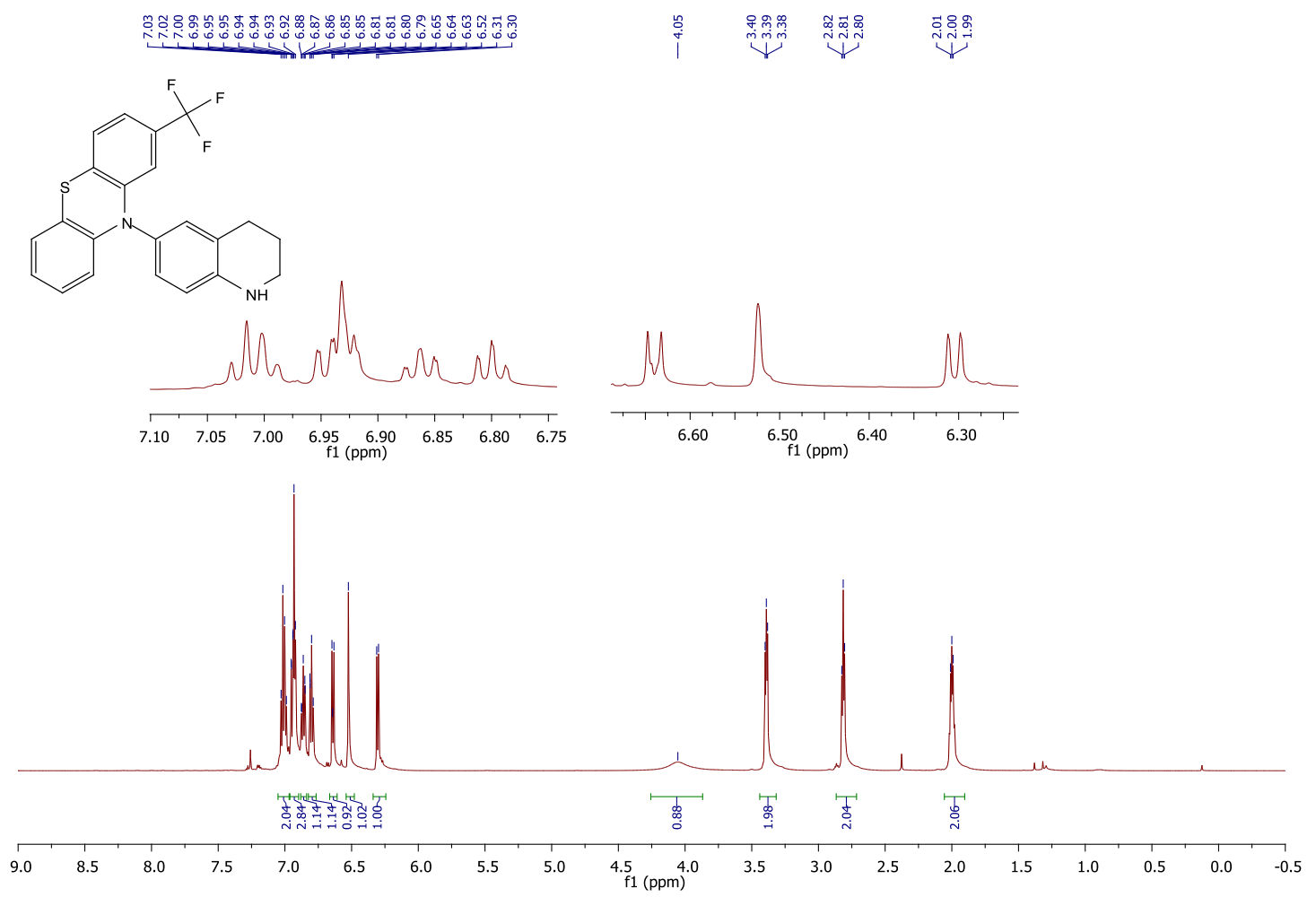

3 ba
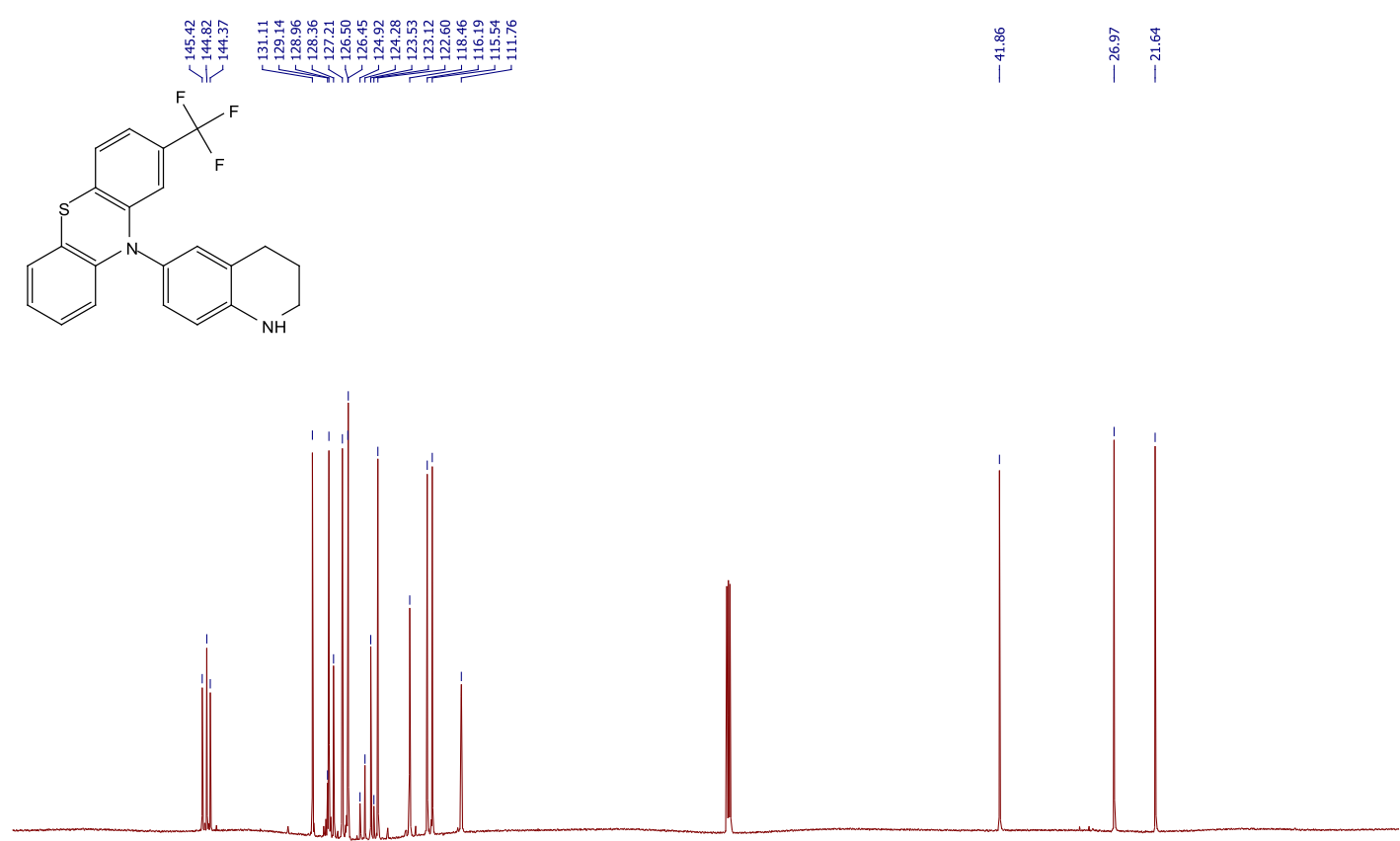

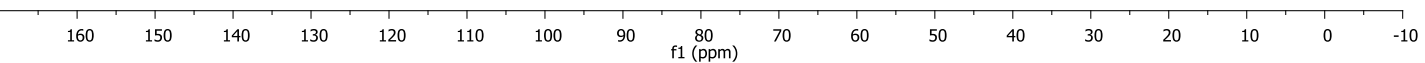


$3 b a$<smiles>FC(F)(F)c1ccc2c(c1)N(c1ccc3c(c1)CCCN3)c1ccccc1S2</smiles>

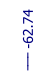

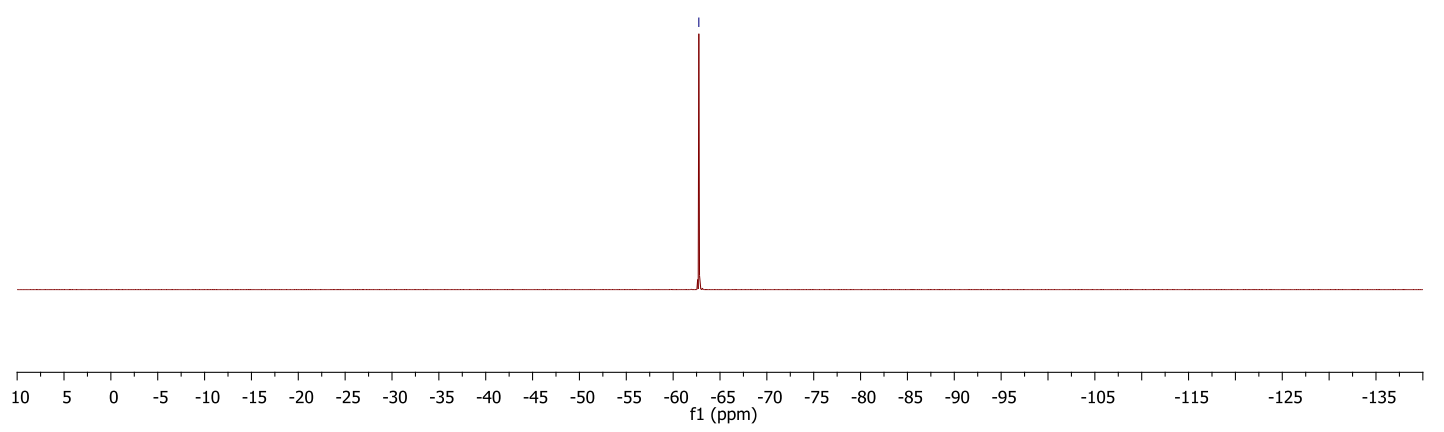




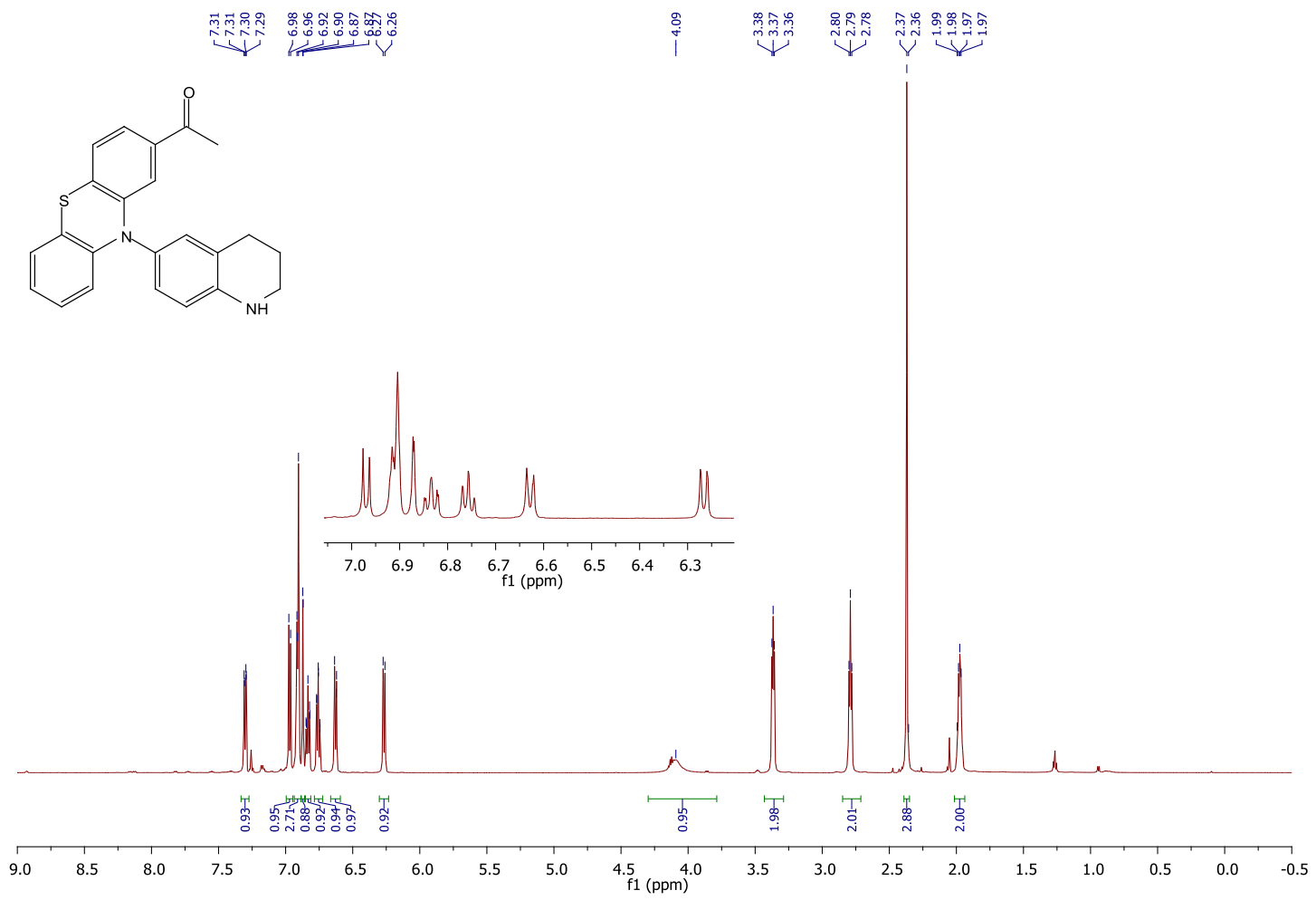

3ca

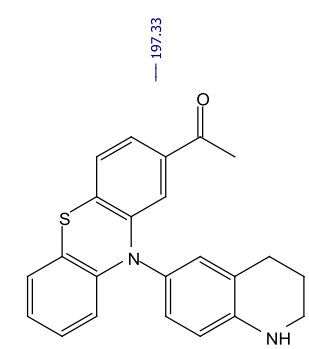

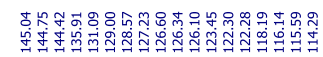

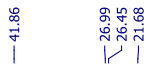

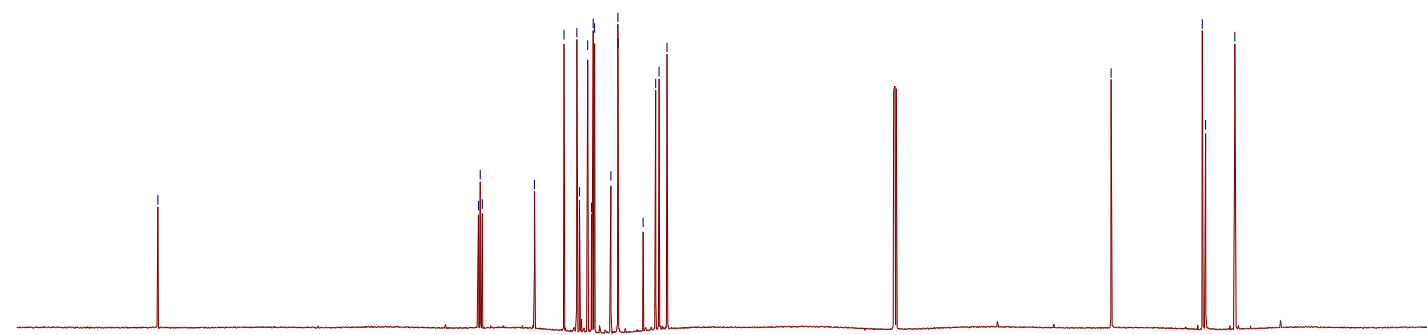

\begin{tabular}{rllllllllllllllllllllllll}
\hline & 220 & 210 & 200 & 190 & 180 & 170 & 160 & 150 & 140 & 130 & 120 & $\underset{\mathrm{f} 1(\mathrm{ppm})}{110}$ & 100 & 90 & 80 & 70 & 60 & 50 & 40 & 30 & 20 & 10 & 0 &
\end{tabular} 


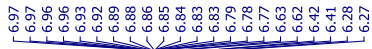

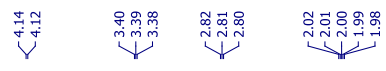<smiles>N#Cc1ccc2c(c1)N(c1ccc3c(c1)CCCN3)c1ccccc1S2</smiles>

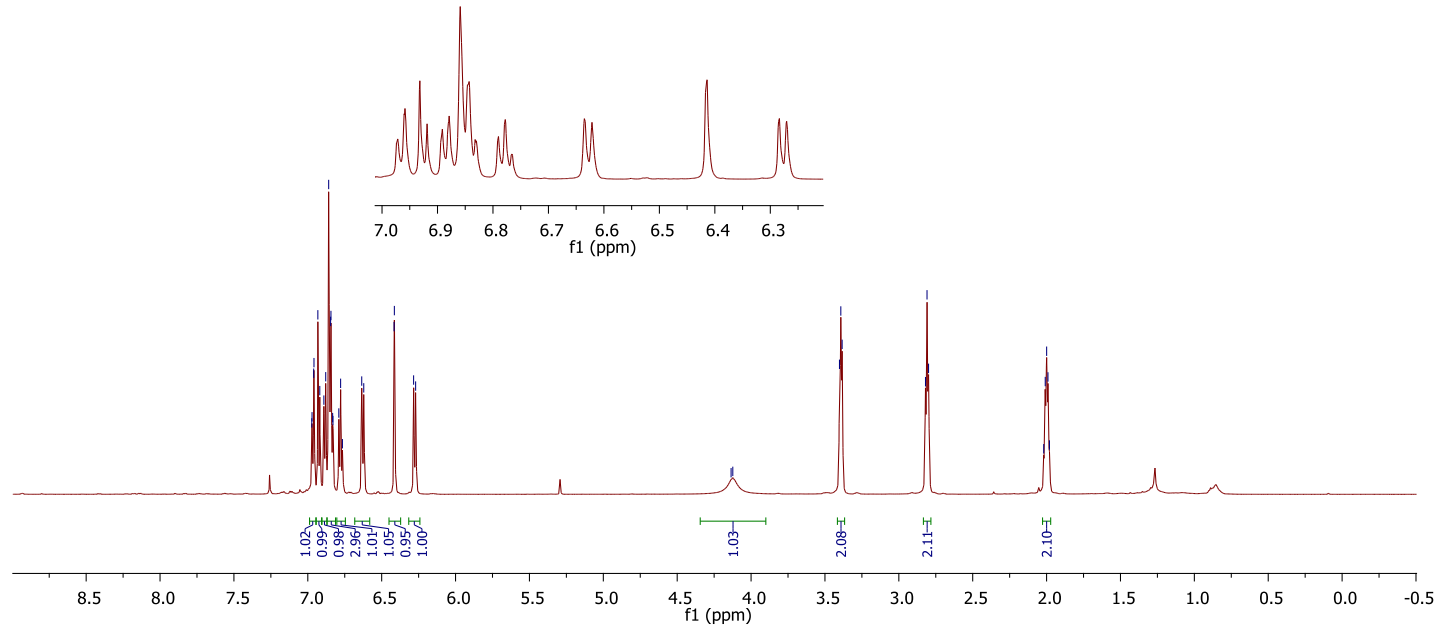

3da

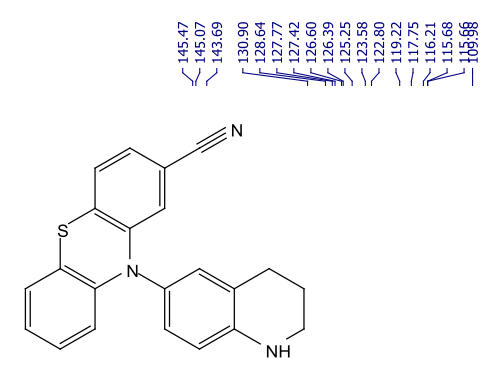

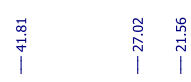

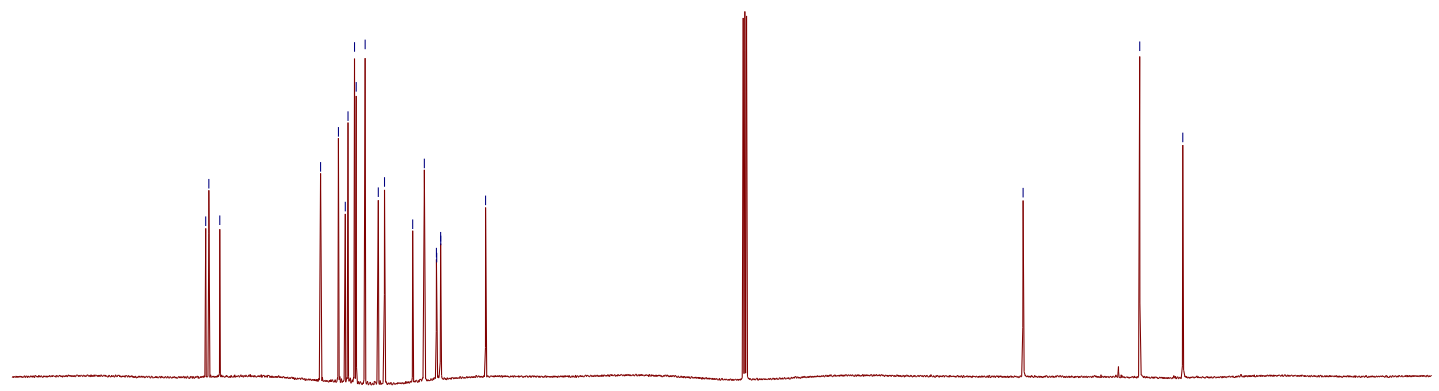

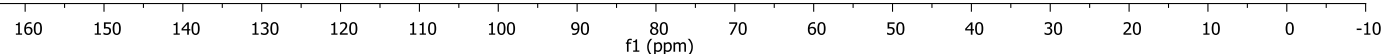


$3 e a$
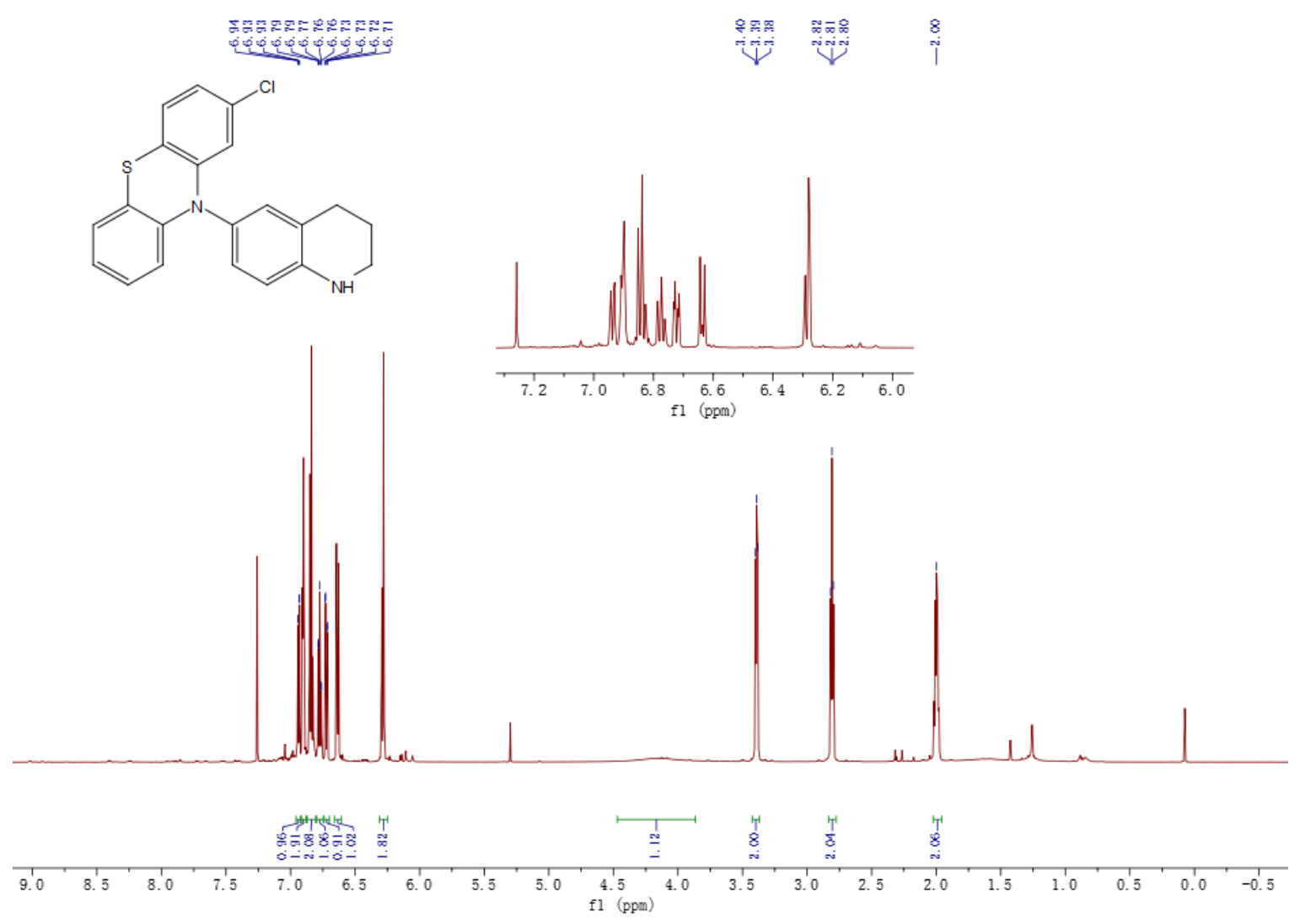

$3 e a$

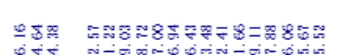

琵
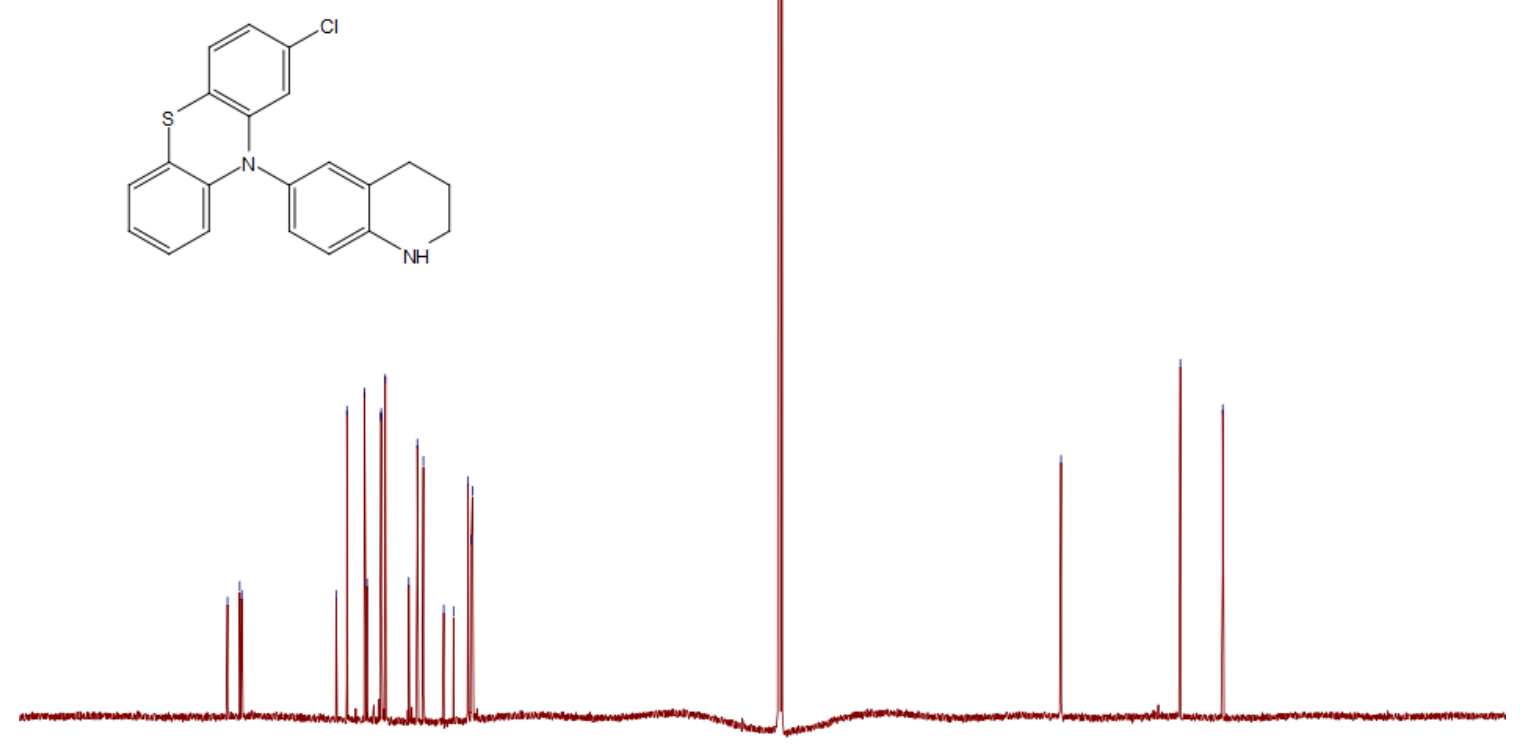

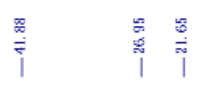

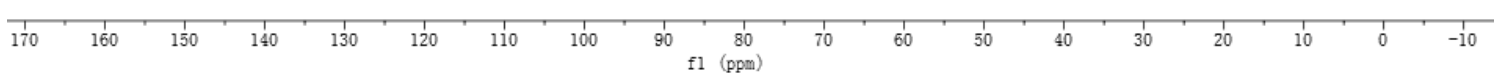

S18 
$3 f a$

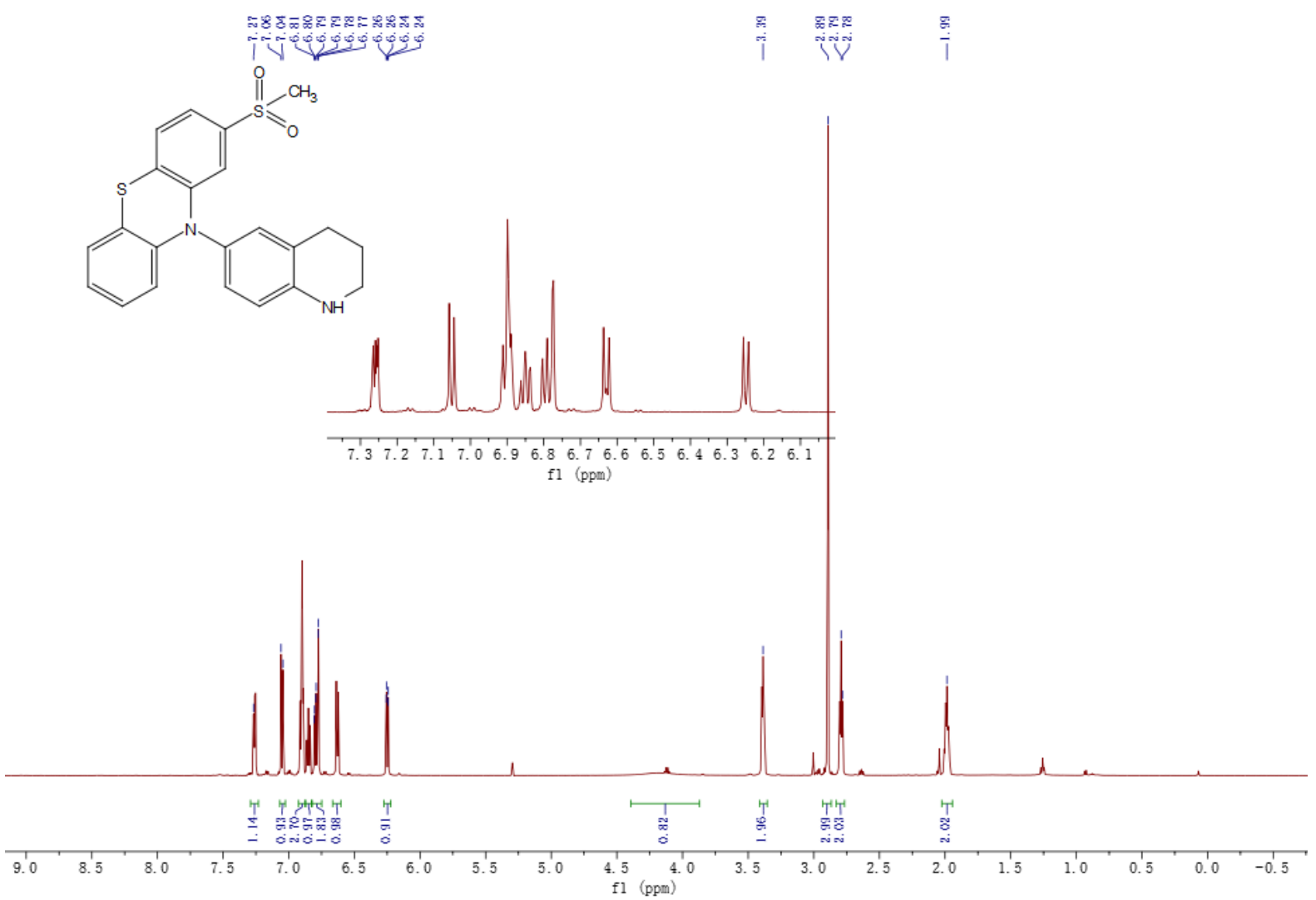

$3 f a$

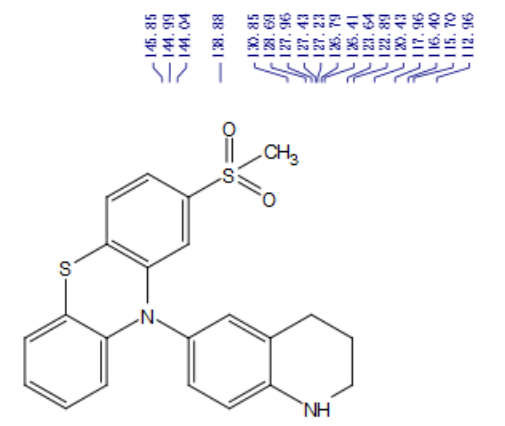

i

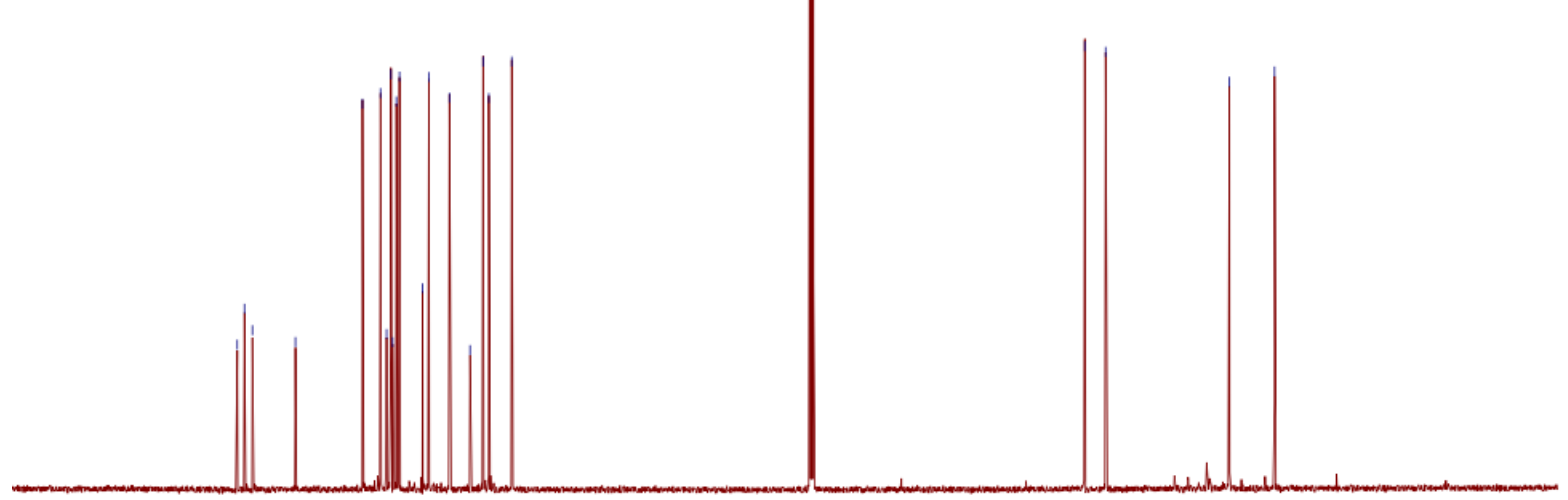

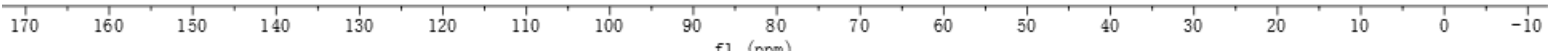




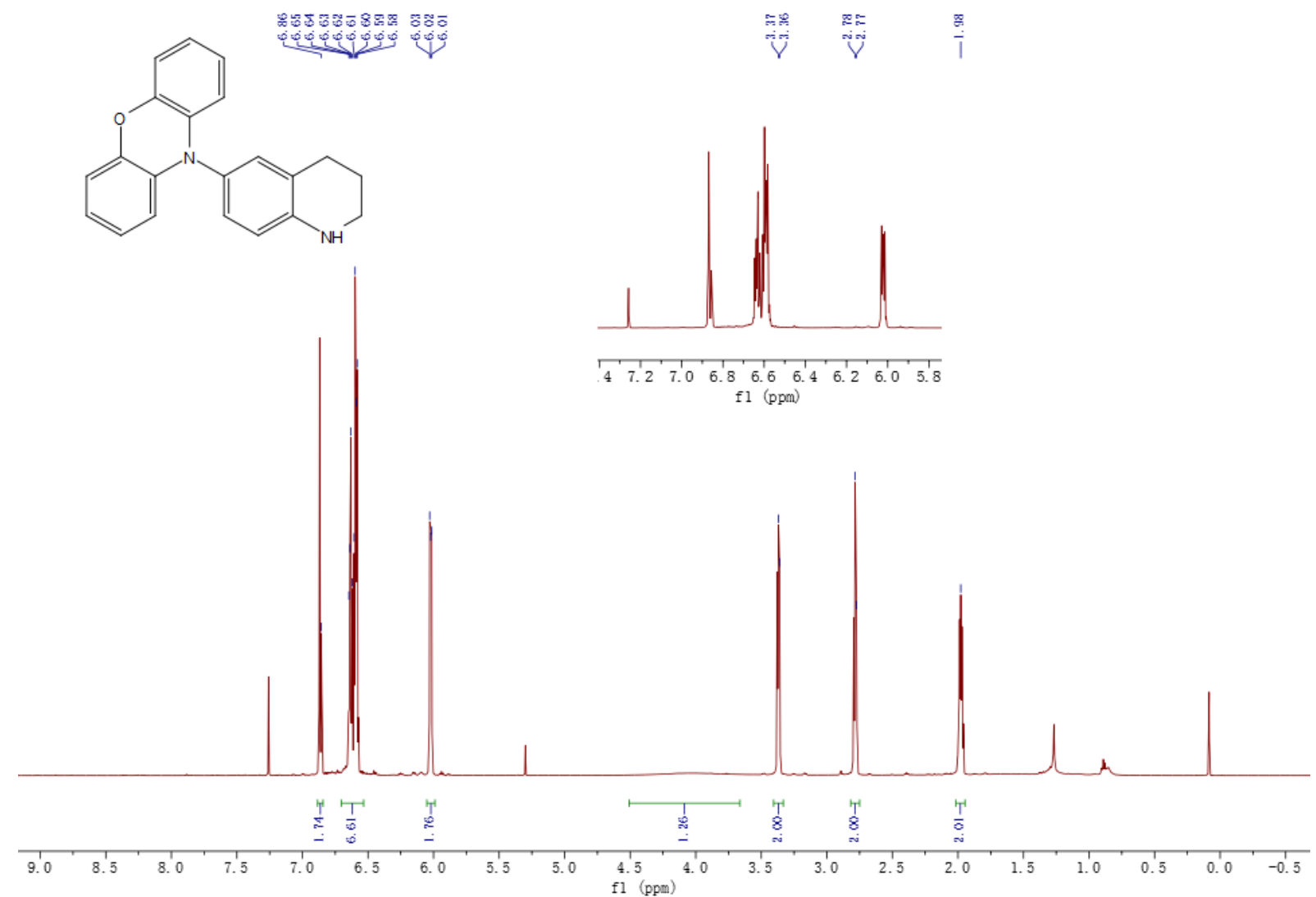

3ga

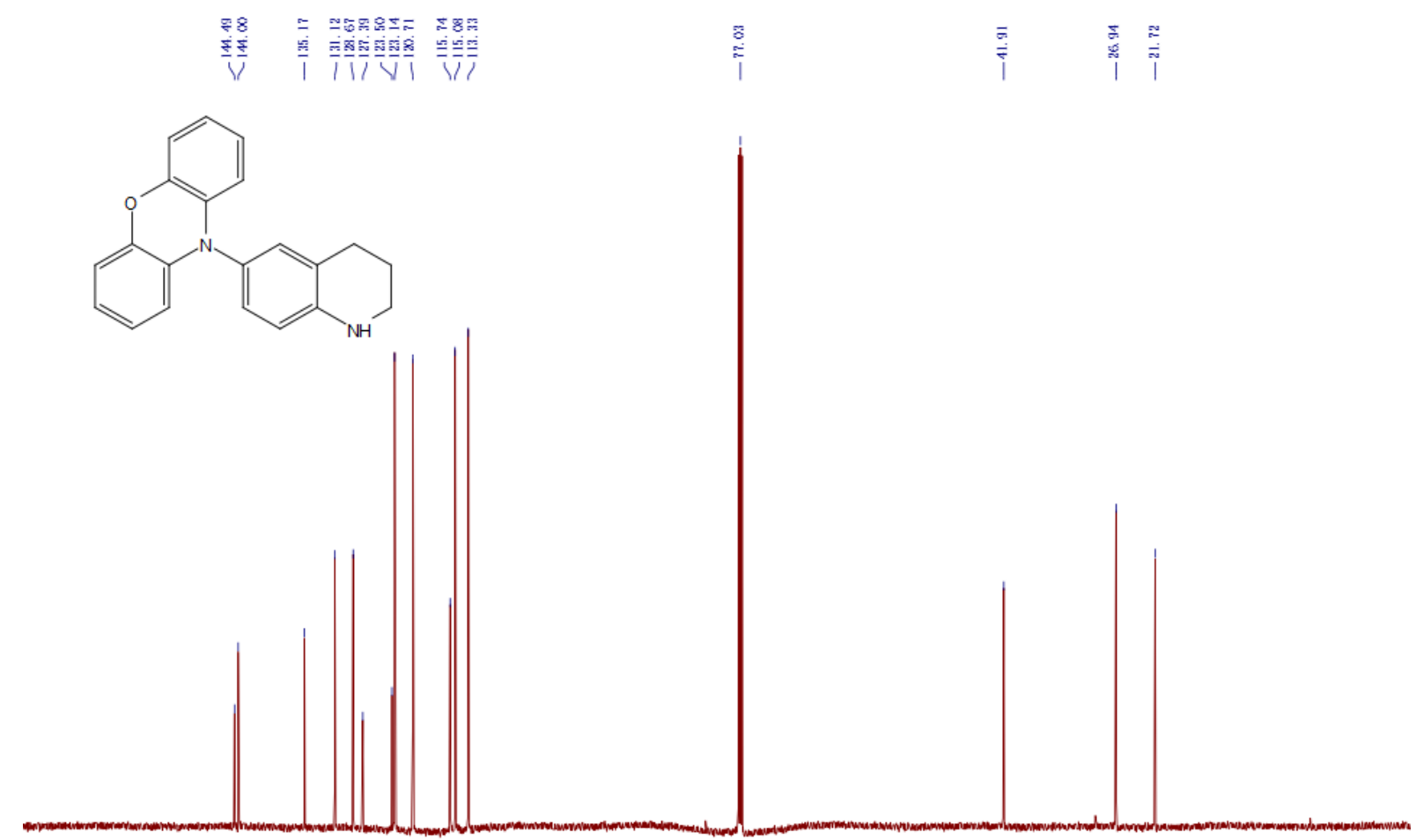

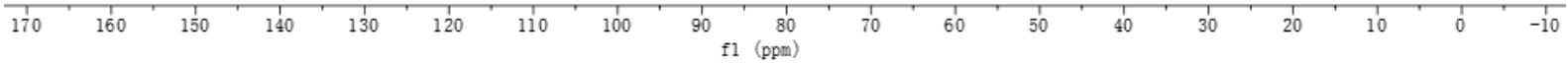


$3 b b$

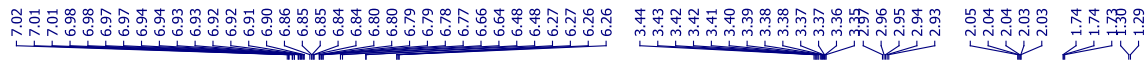

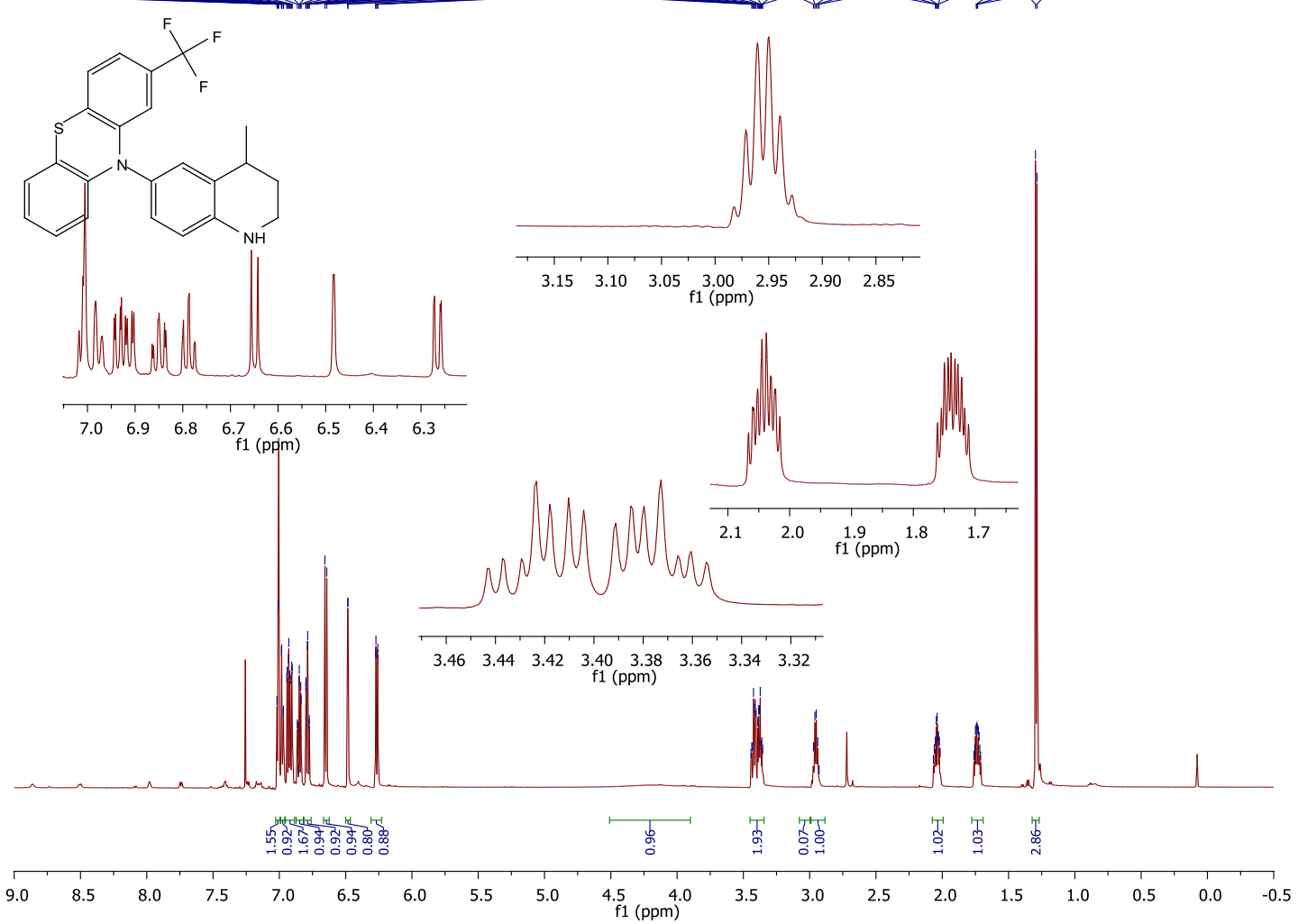

$3 b b$

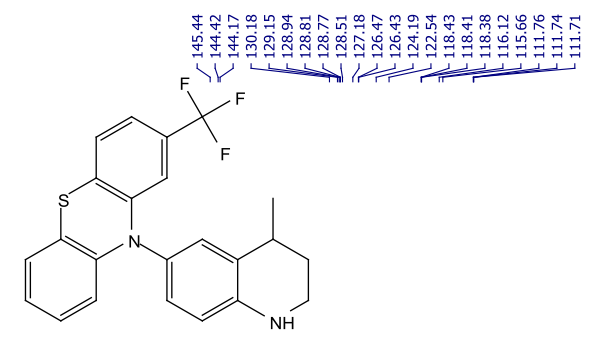

¿ั0

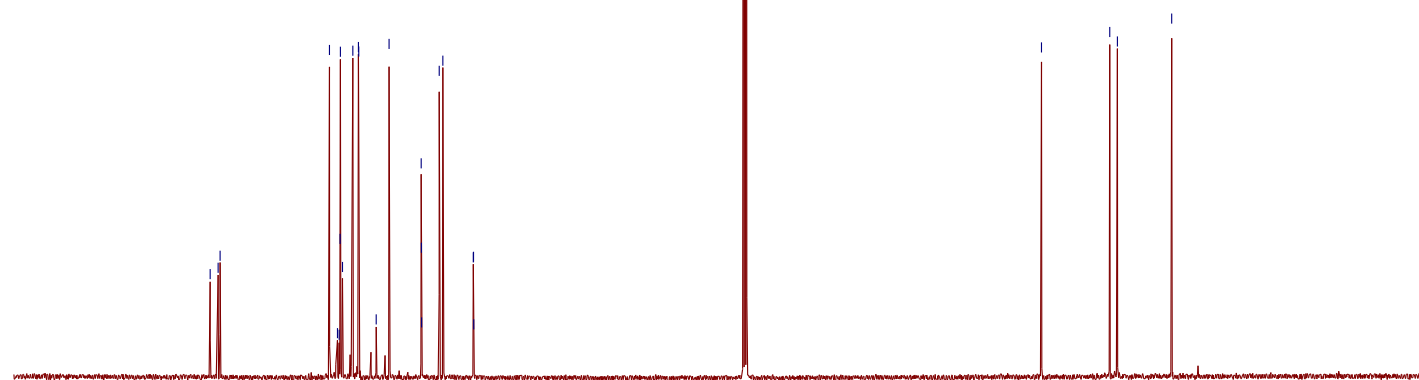

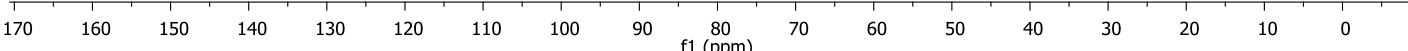


$3 b b$

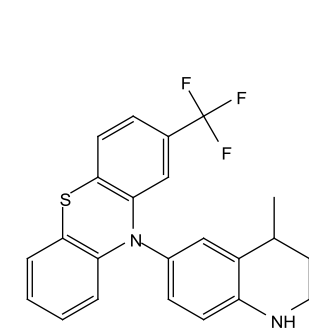

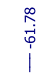

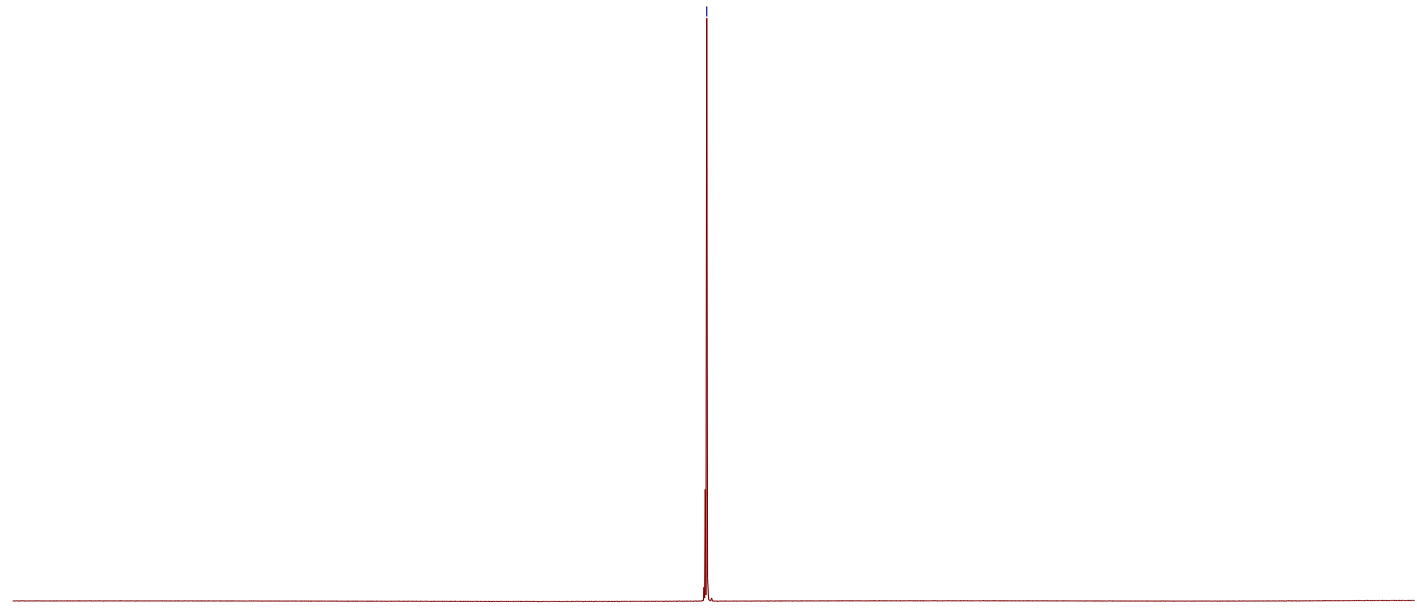

T.

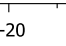

$-30$

f1 (ppm)

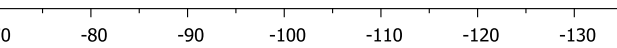


$3 c b$

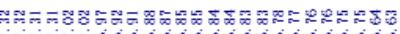
ปัن

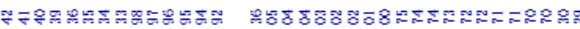

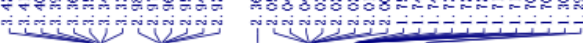<smiles>CC(=O)c1ccc2c(c1)N(c1ccc3c(c1)C(C)CCN3)c1ccccc1S2</smiles>

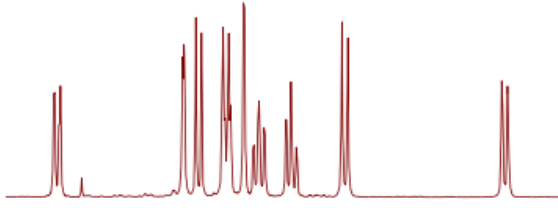

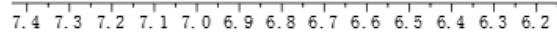
fl $(\mathrm{ppm})$

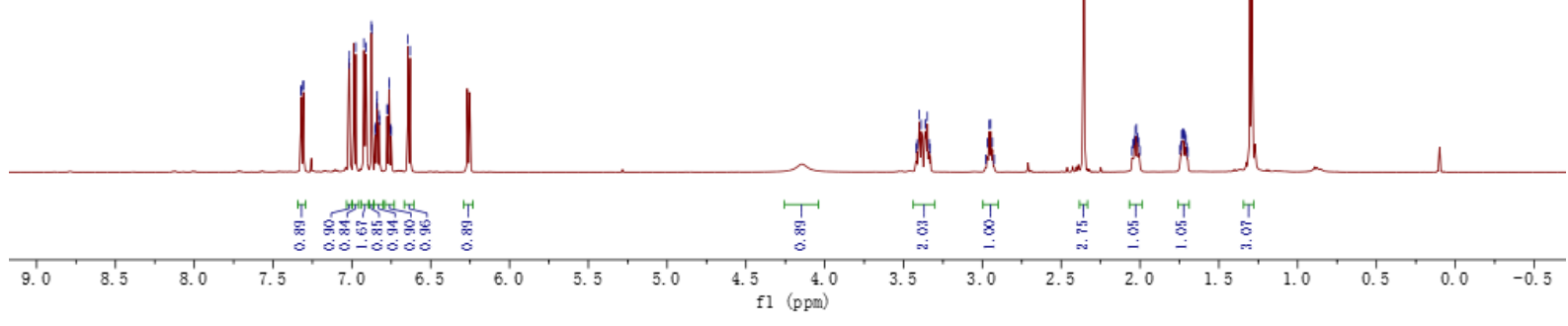

$3 c b$

$$
\frac{1}{20}
$$

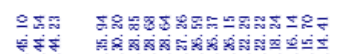

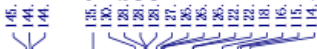<smiles>CC(=O)c1ccc2c(c1)N(c1ccc3c(c1)C(C)CCN3)c1ccccc1S2</smiles>

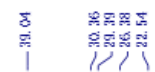

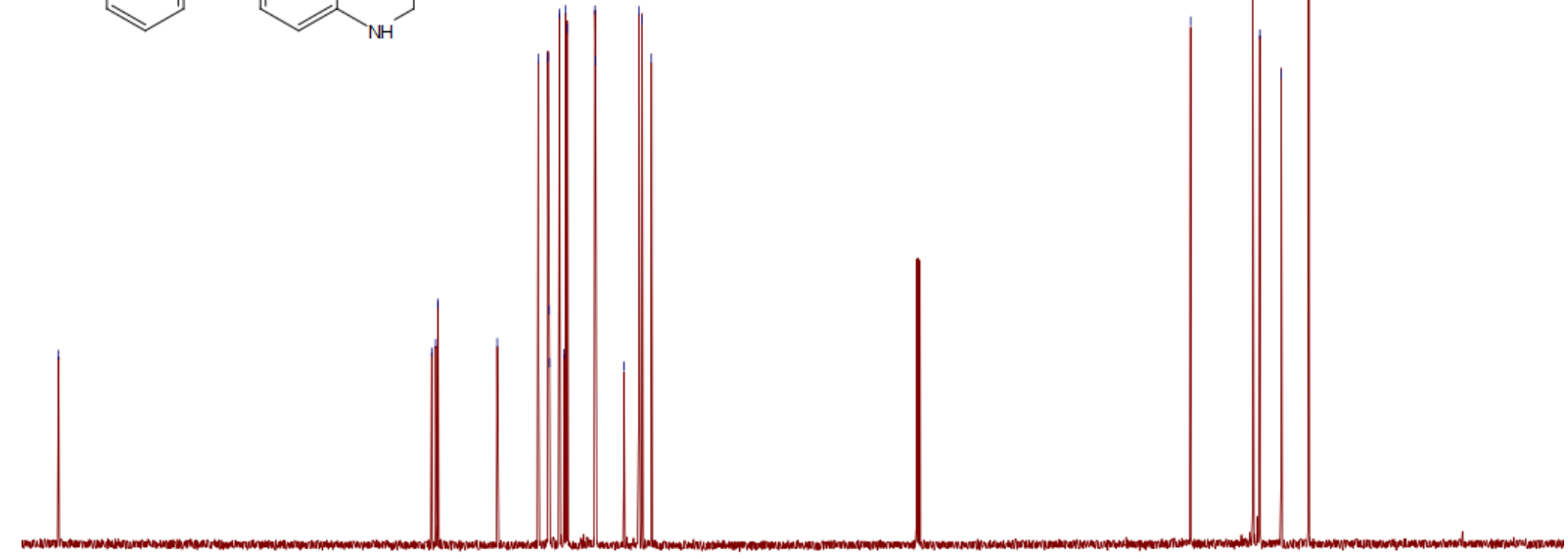


$3 d b$

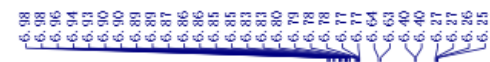

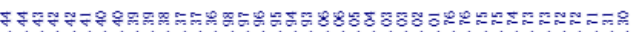

Fis<smiles>CC1CCNc2ccc(N3c4ccccc4Sc4ccc(C#N)cc43)cc21</smiles>

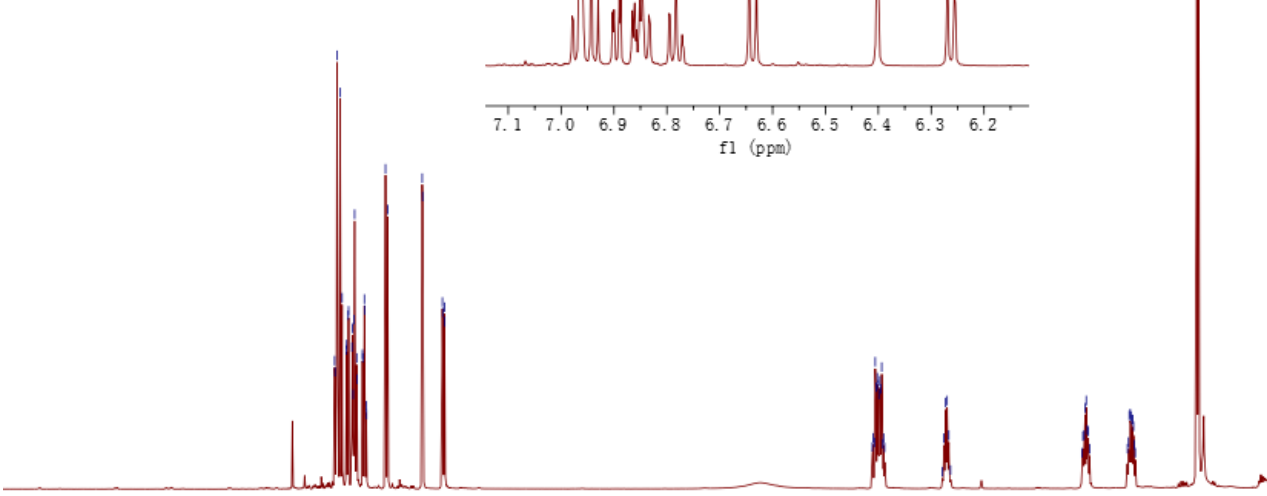

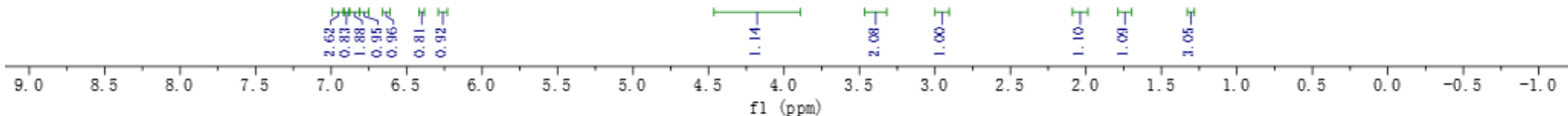

$3 d b$

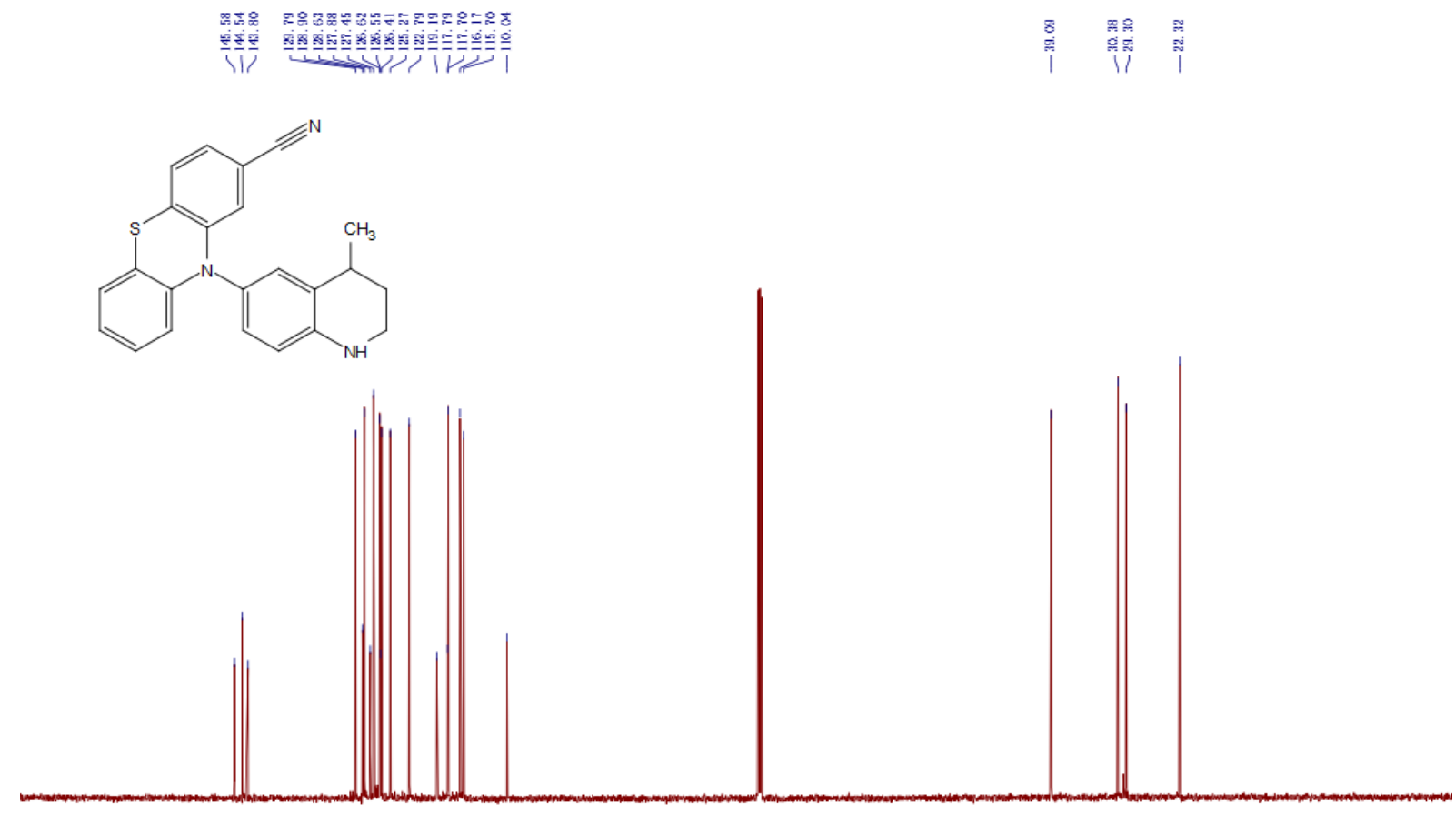

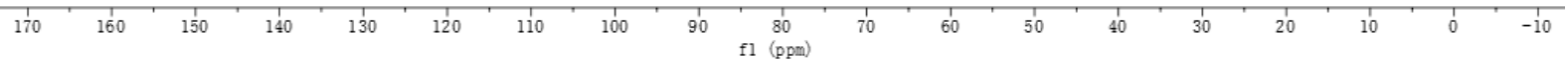


3eb

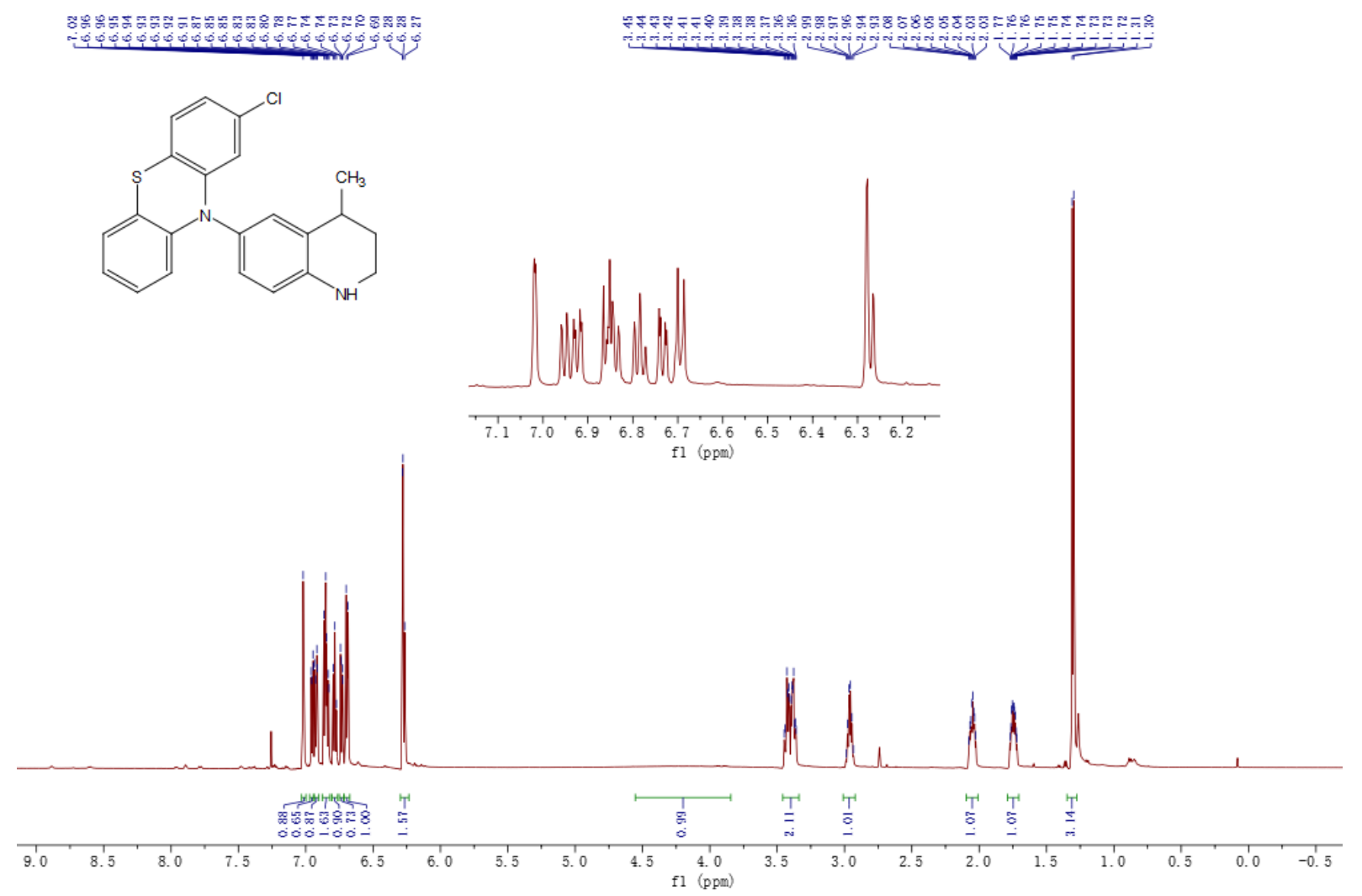

3eb

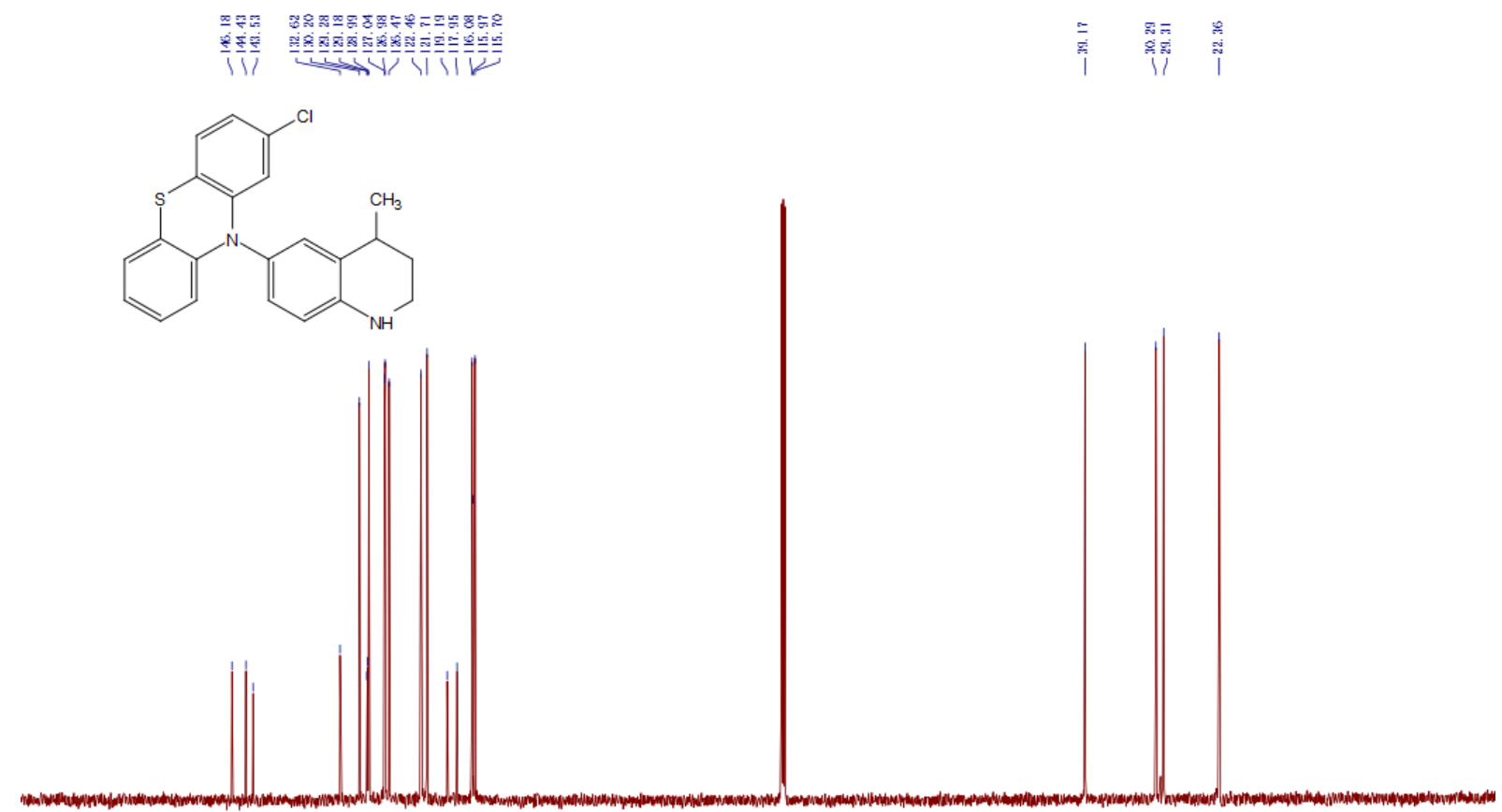

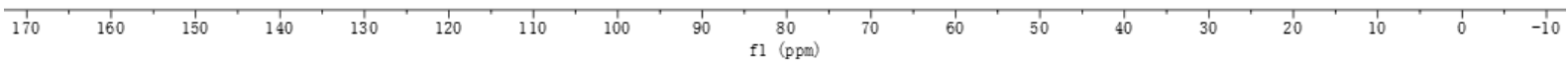


$3 b c$

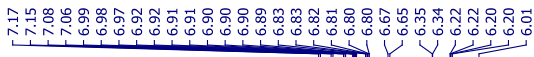

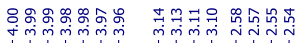

$\sqrt[7]{\mathcal{Z}}$<smiles>CC1Cc2cc(N3c4ccccc4Sc4ccc(C(F)(F)F)cc43)ccc2N1</smiles>
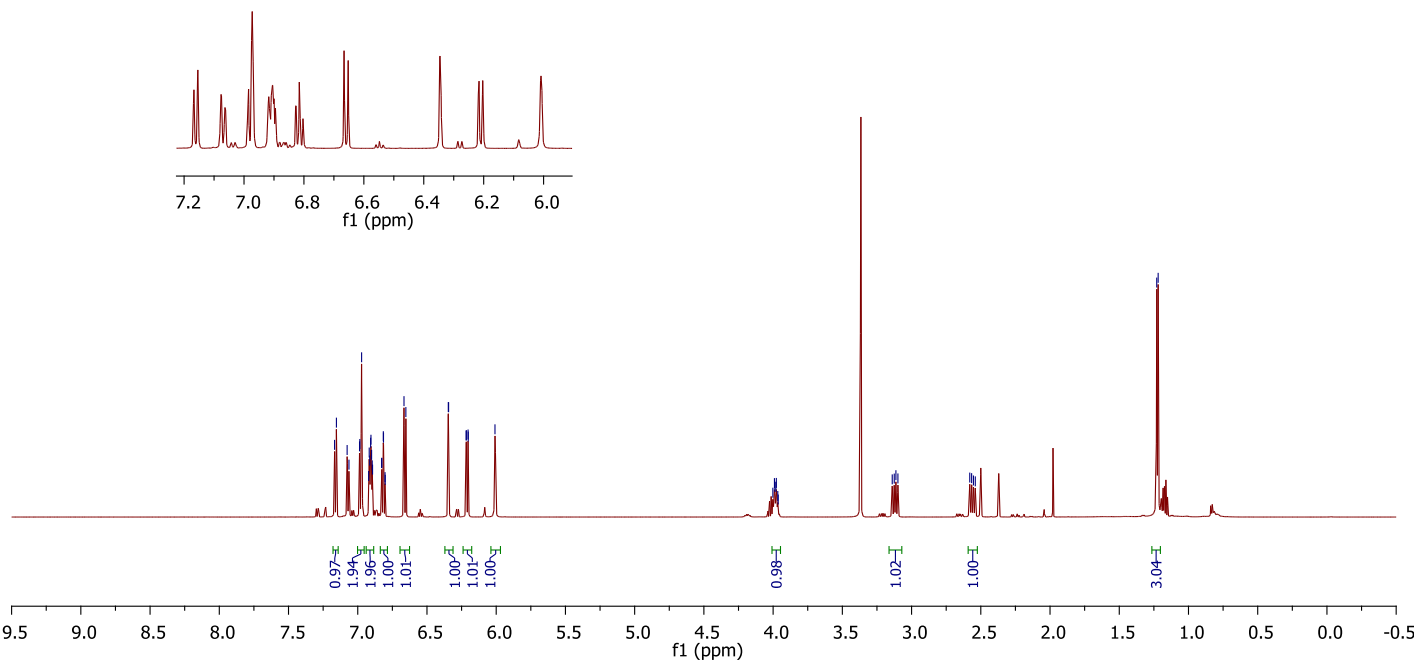

$3 b c$

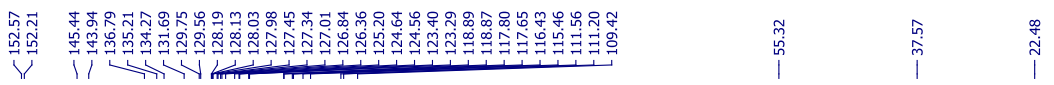
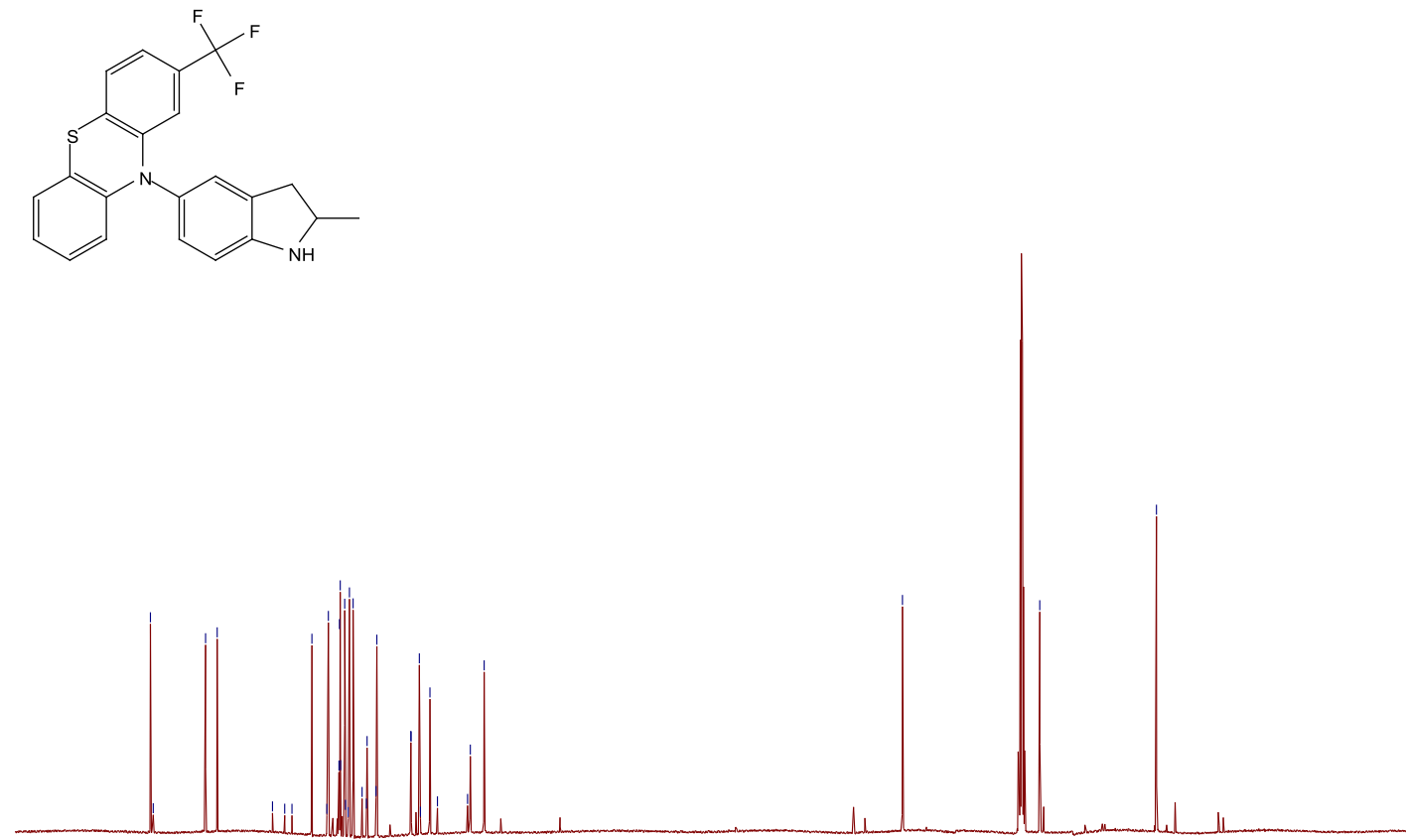

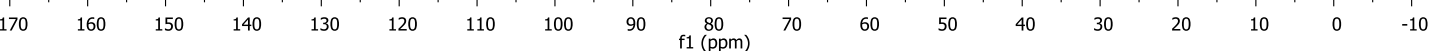


$3 b c$

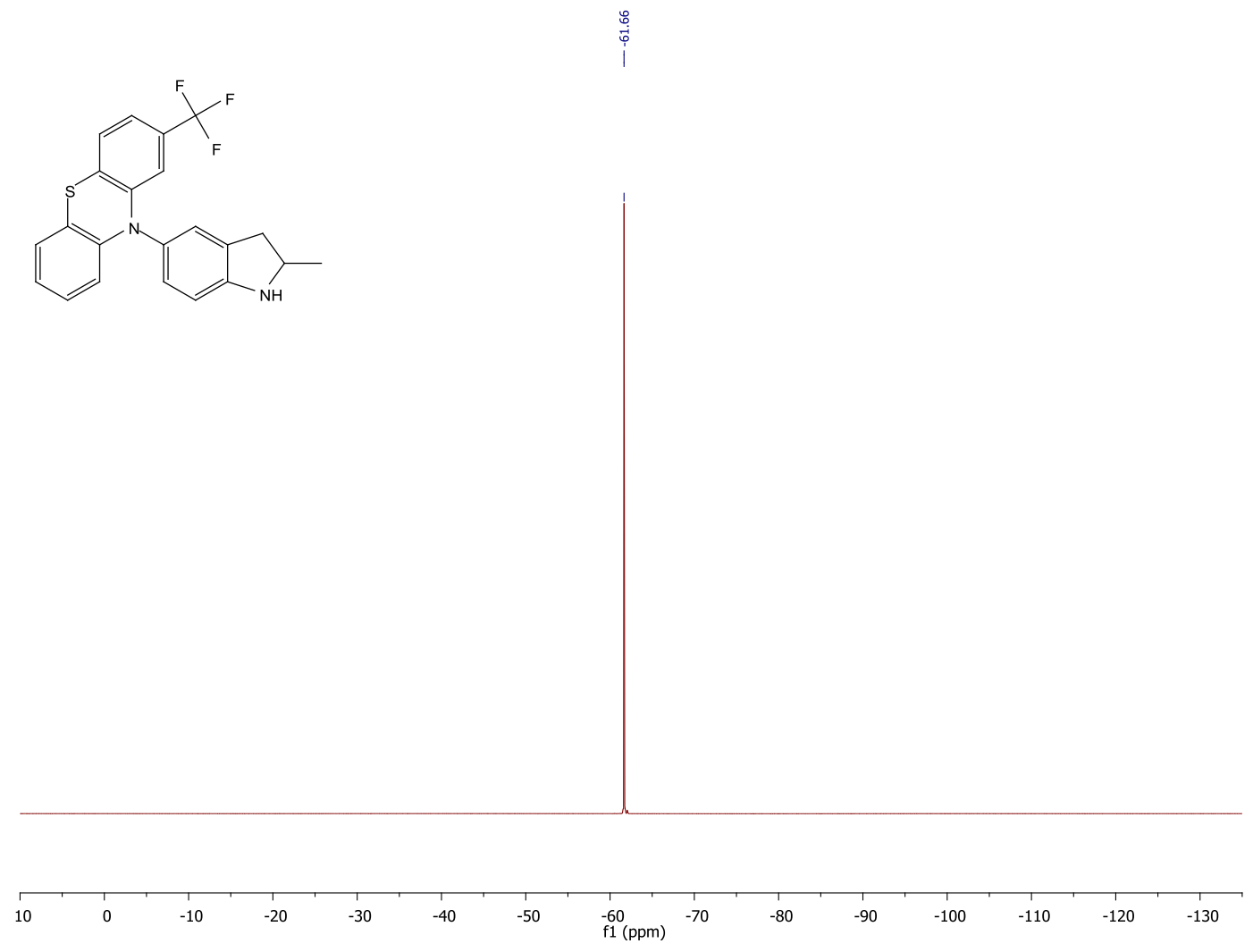


诲<smiles>CC(=O)c1ccc2c(c1)N(c1ccc3c(c1)CC(C)N3)c1ccccc1S2</smiles>
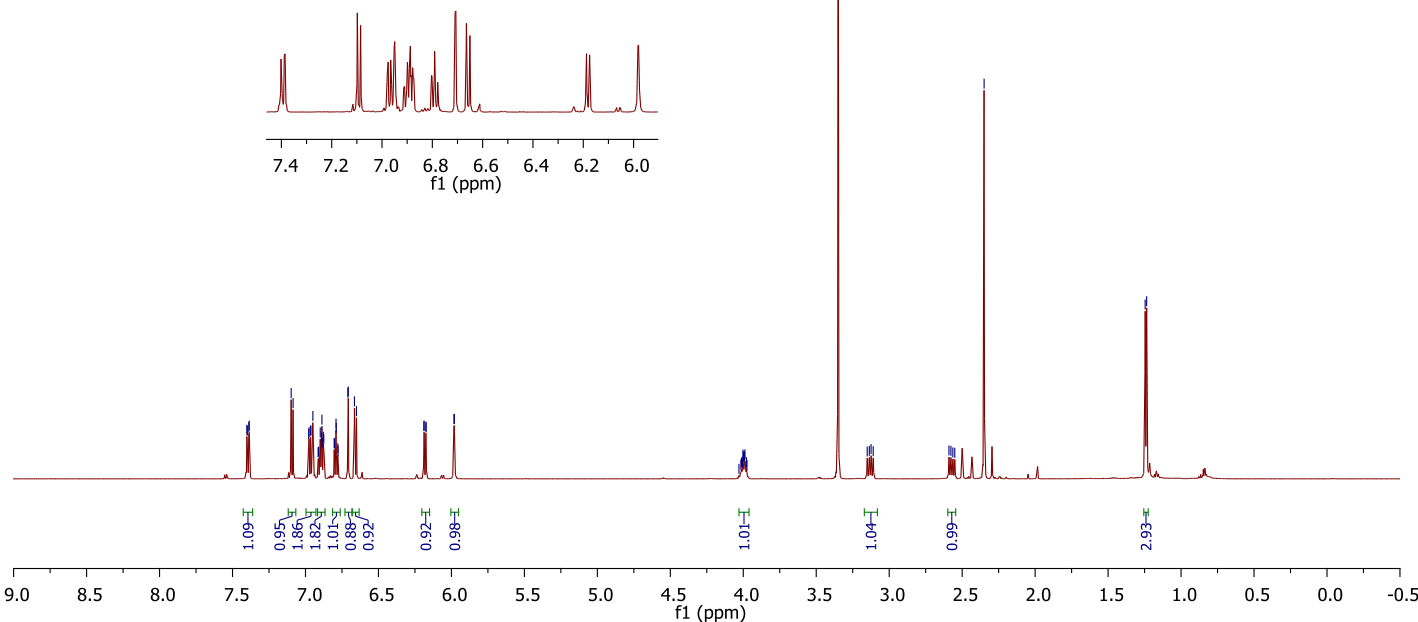

3cc<smiles>CC(=O)c1ccc2c(c1)N(c1ccc3c(c1)CC(C)N3)c1ccccc1S2</smiles>

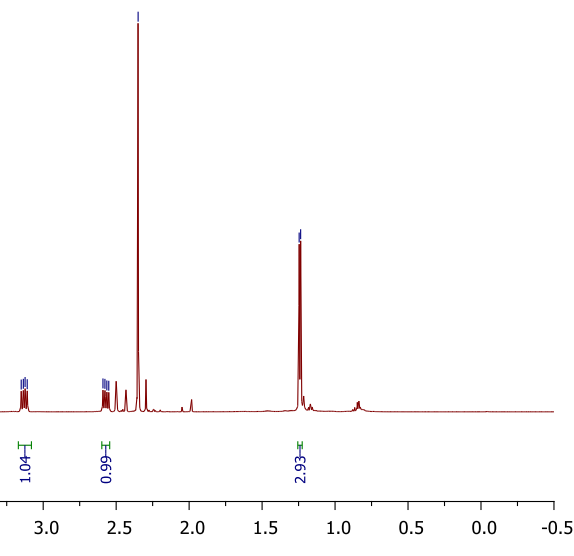


$3 d c$

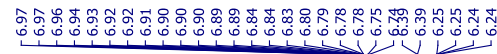

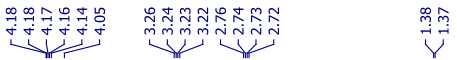
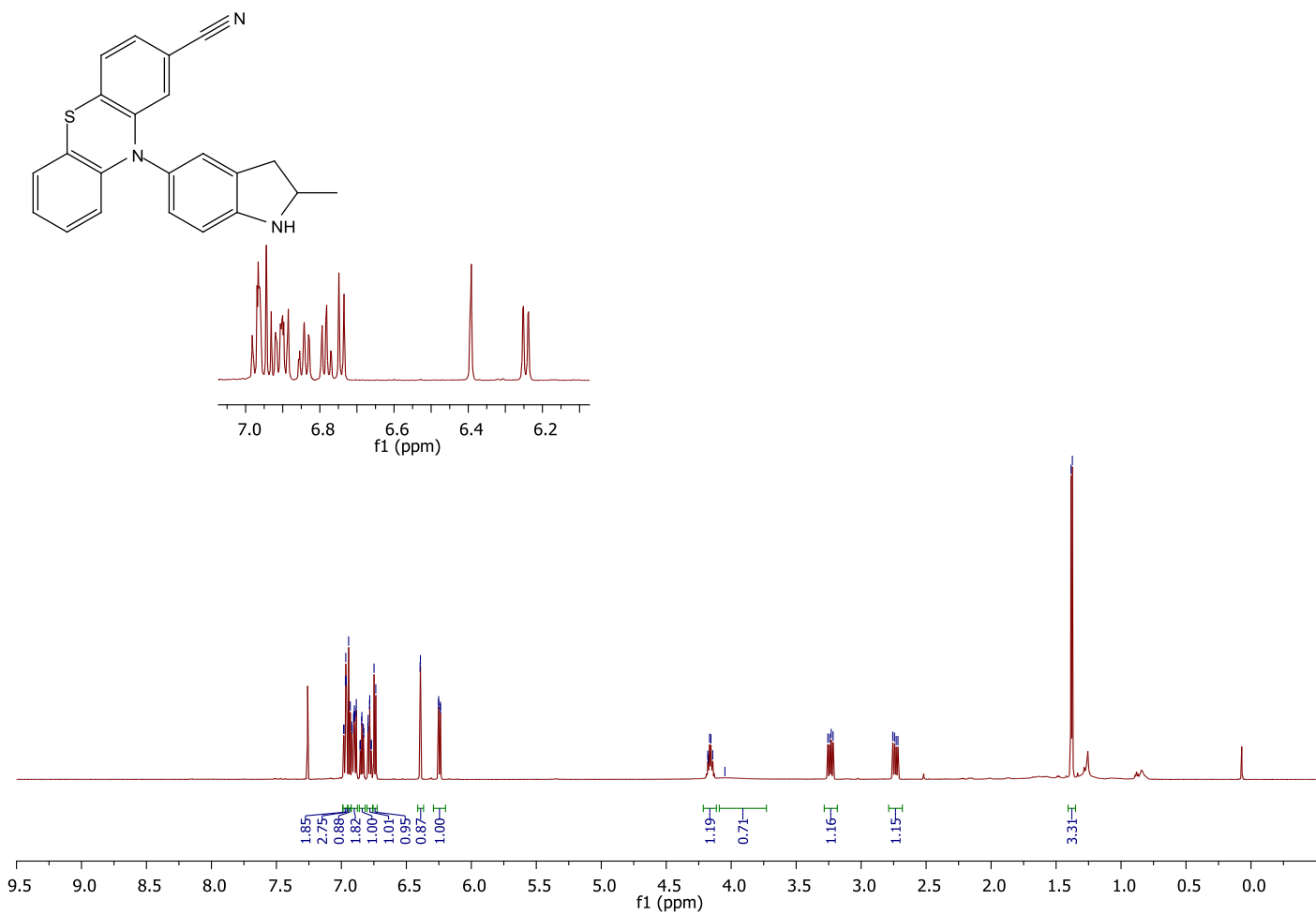

3dc

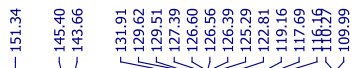

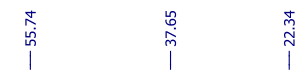
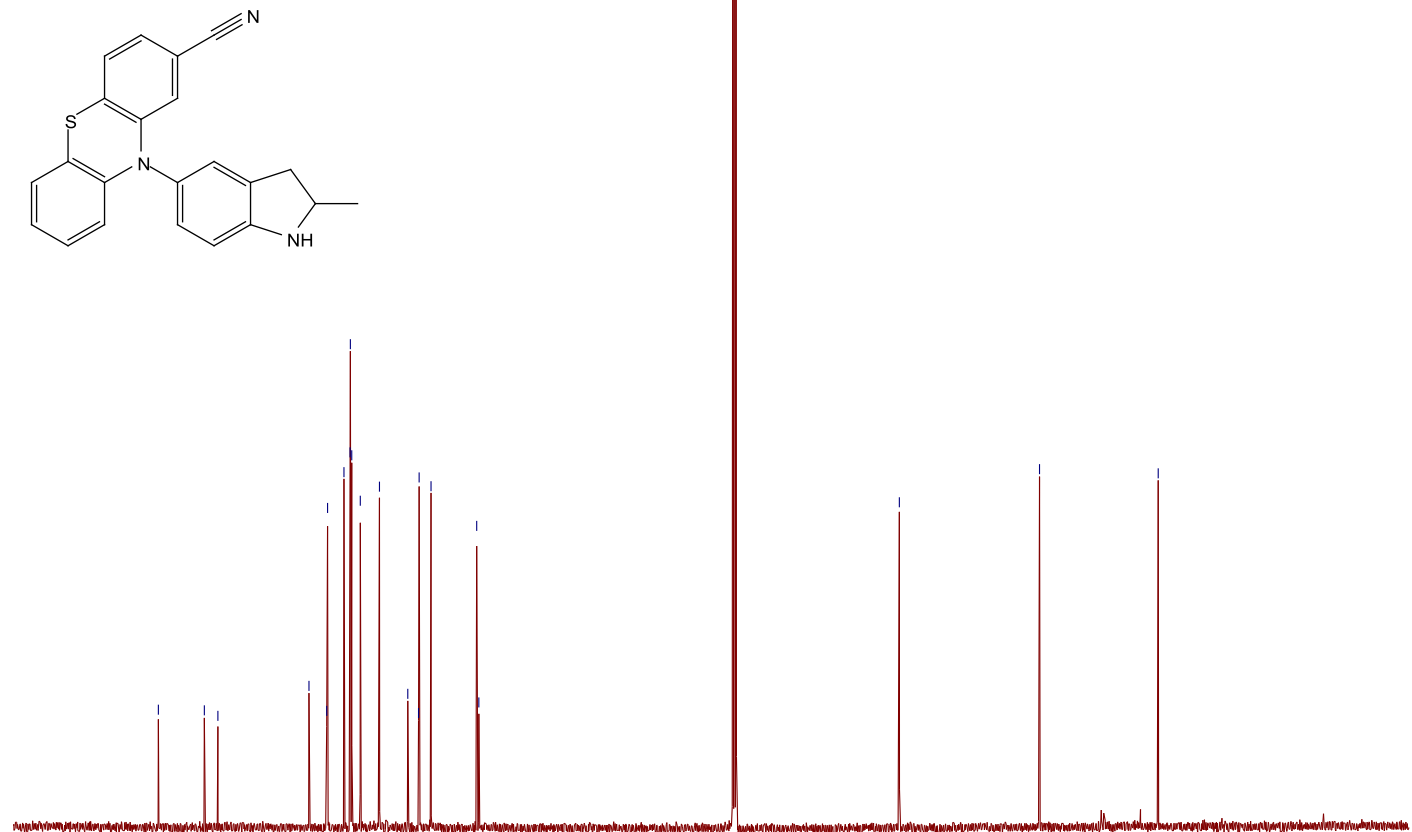

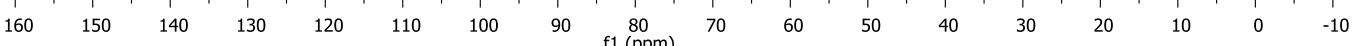




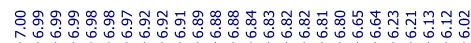
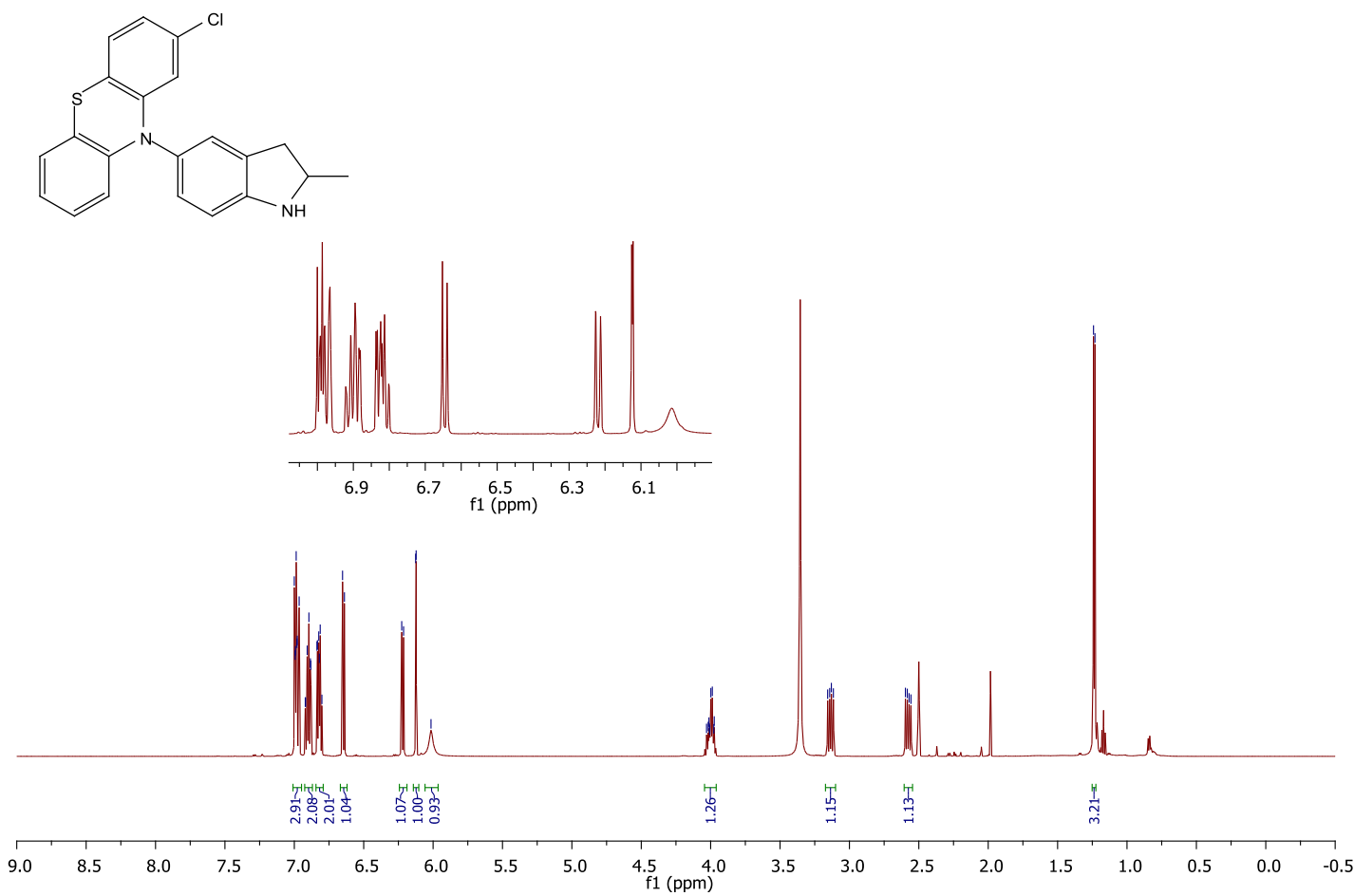

$3 e c$
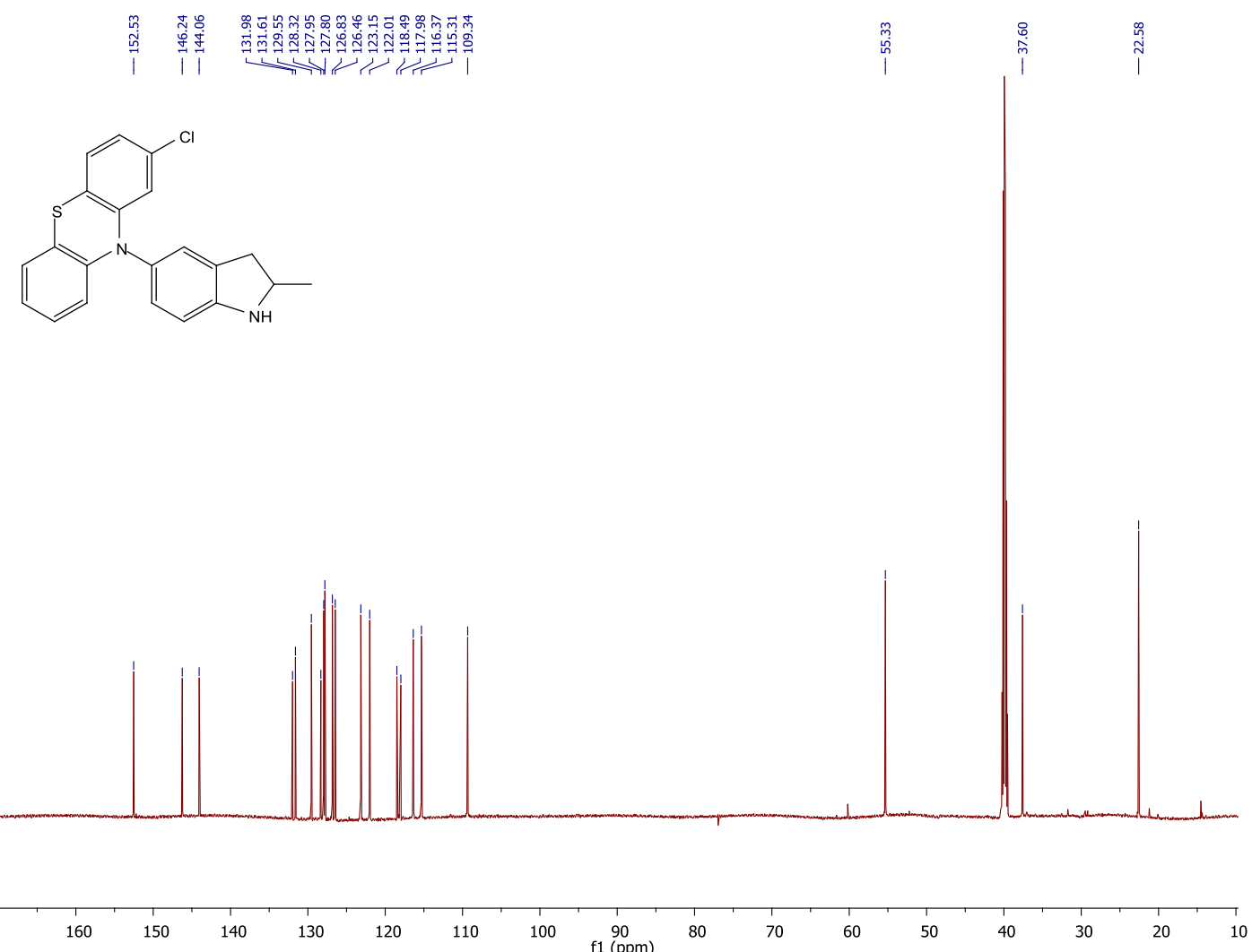
$4 c c$

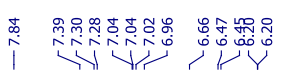

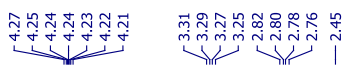

$\sqrt[\vec{m}]{\mathrm{Y}}$
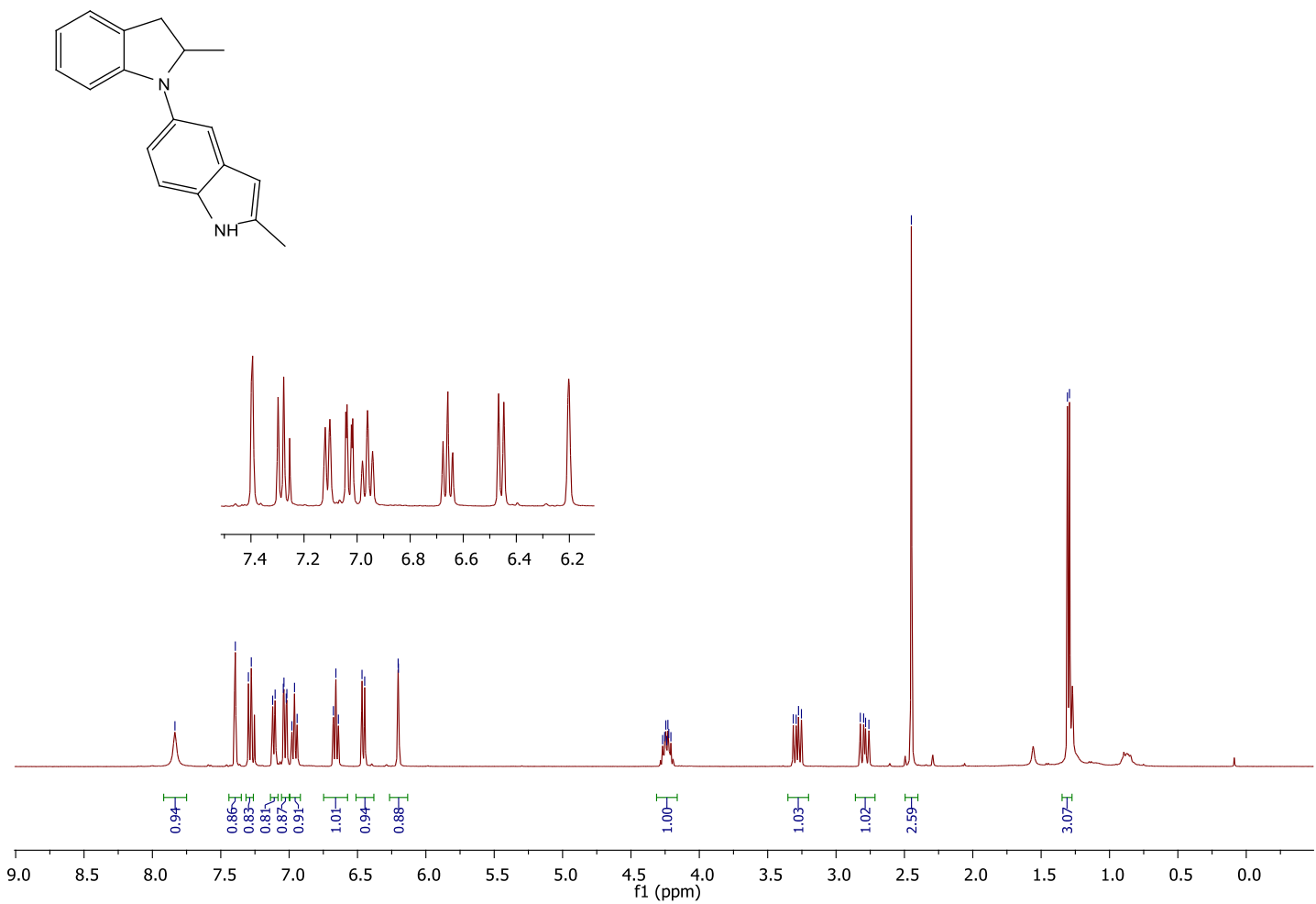

$4 c c$
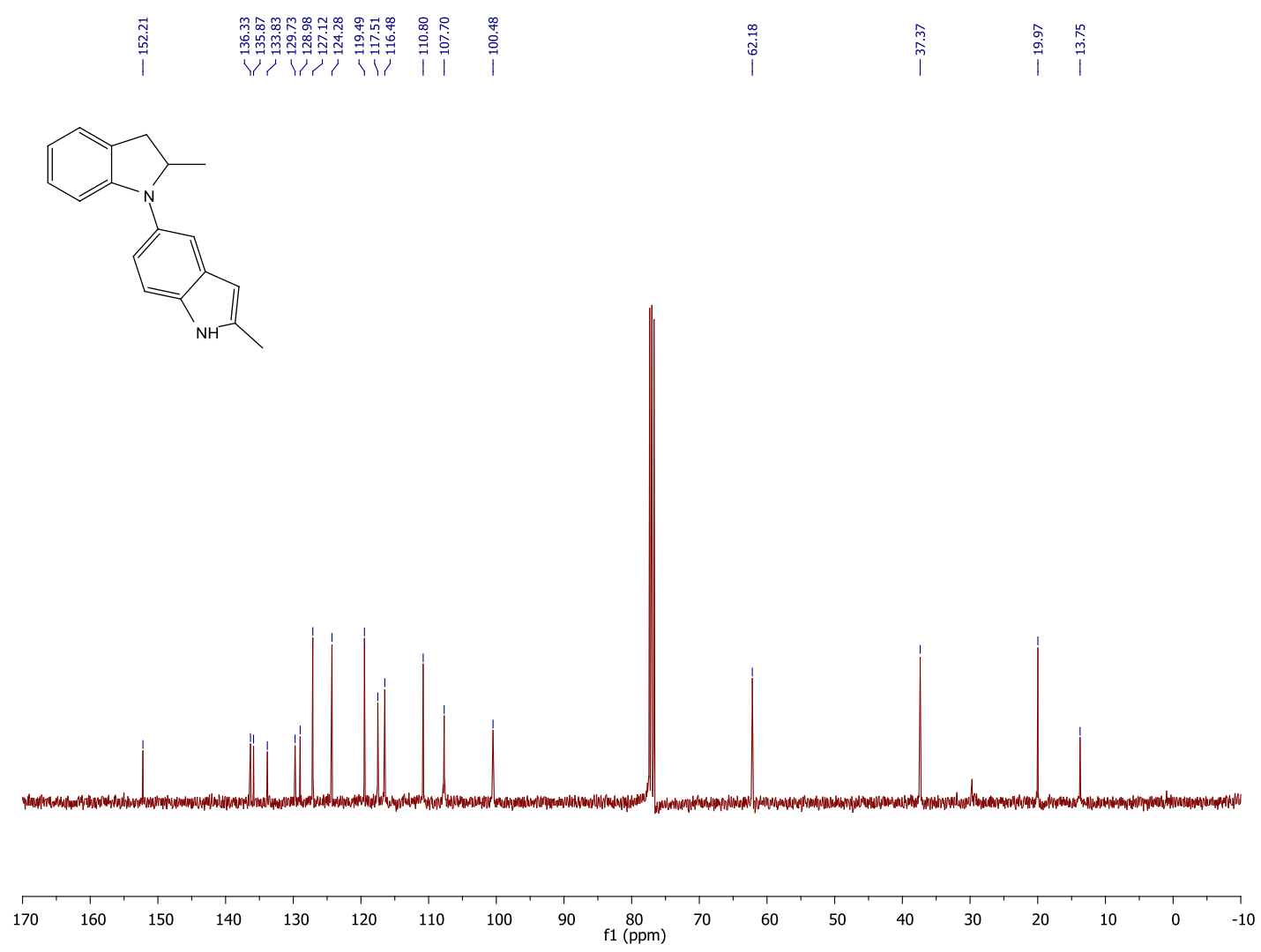

S31 
$4 c c(\cos Y)$

$\mathrm{NH}$ is on the indole side

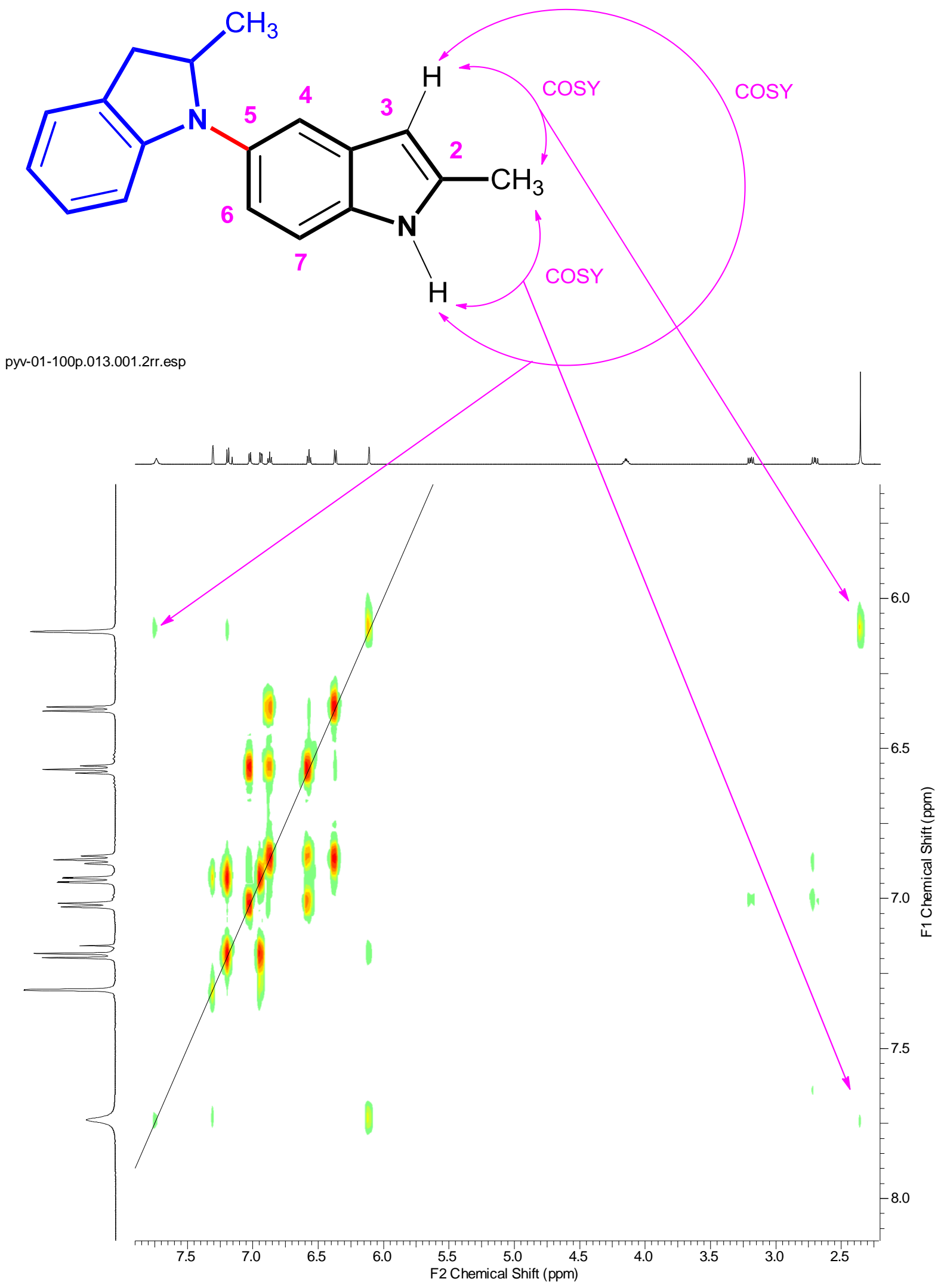




\section{Zoom in:}

pyv-01-100p.013.001.2rr.esp
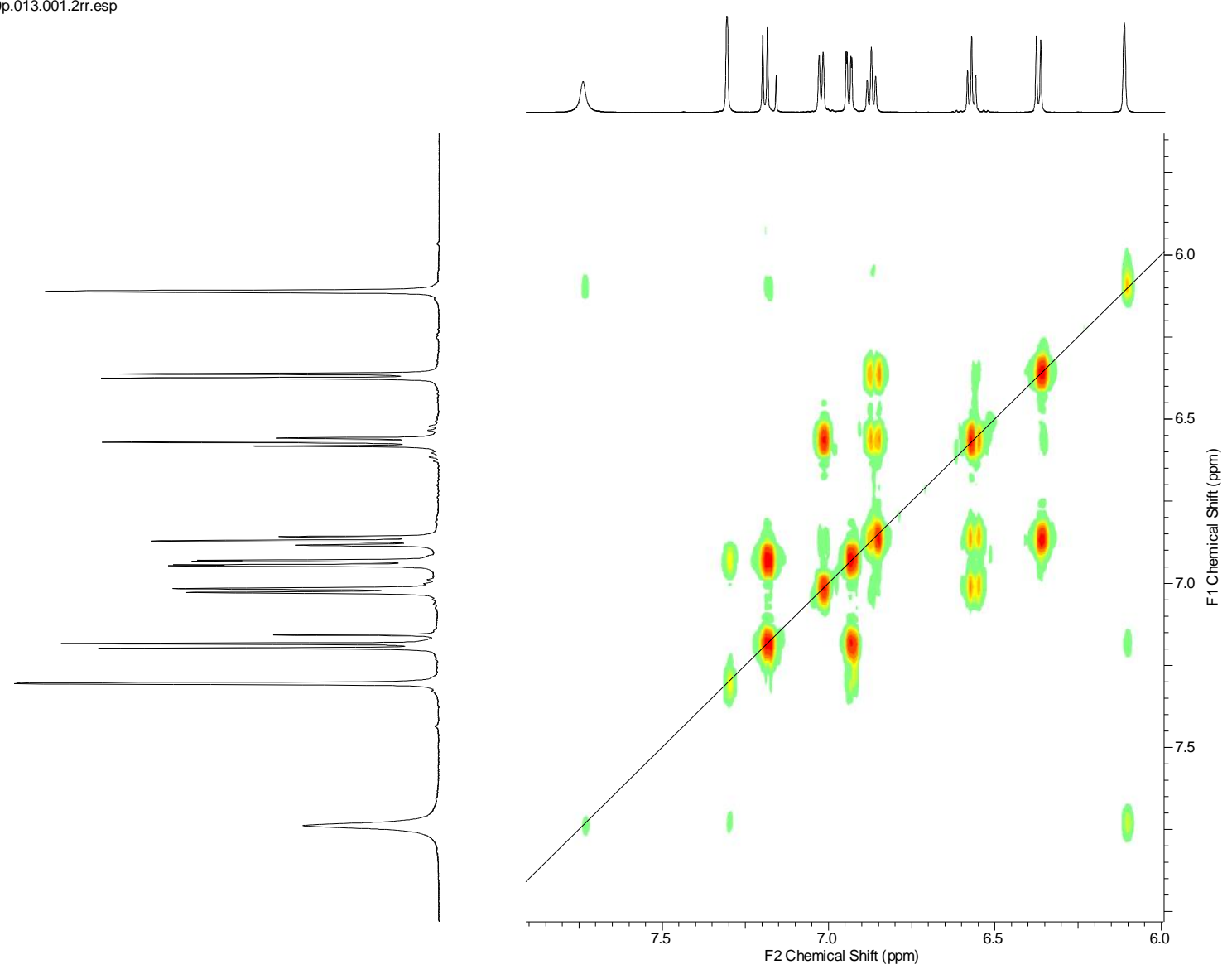